Moroccan J. of Pure and Appl. Anal. (MJPAA)

Volume 5(2), 2019, Pages 140-163

ISSN: Online 2351-8227 - Print 2605-6364

DOI 10.2478/mjpaa-2019-0011

\title{
Fixed Point Theorems in Complex Valued Extended $b$-Metric Spaces
}

\author{
Naimat Ullah ${ }^{1, a}$, Mohammed Shehu Shagari ${ }^{2, b}$ and Akbar Azam ${ }^{1, c}$
}

Aвstract. In this article, inspired by the concepts of extended $b$-metric spaces, we introduce the notion of complex valued extended $b$-metric spaces. Using this new idea, some fixed point theorems involving rational contractive inequalities are proved. The established results herein augment several significant work in the comparable literature.

2010 Mathematics Subject Classification. 46S40, 47H10, 54H25.

Key words and phrases. Extended $b$-metric space; Multi-valued mapping; Fixed point; Complex valued metric space.

\section{Introduction and Preliminary}

Fixed point theory is a well known and vast field of research in mathematical sciences. This field is known as the mixture of analysis which includes topology, geometry and algebra. In particular, fixed point technique is a central tool in the study of non-linear analysis. In this area, a huge involvement has been made by Banach [10], who gave the notion of contraction mapping due to a complete metric space to locate fixed point of the specified function. The classical fixed point theorem due to Banach [10] has been generalized by many researchers in various ways (see, for example, [7, 5, 13, 17]) and the references therein. One may also consult Rhoades [24] for different definitions of contractive type mappings.

In 1969, Nadler [23] initiated the study of fixed point theorems for multi-valued mappings. Nadler's contraction principle encouraged many researchers and as a result, the idea has been developed in different directions

Received July 25, 2019 - Accepted October 18, 2019.

(C) The Author(s) 2017. This article is published with open access by Sidi Mohamed Ben Abdallah University.

${ }^{1}$ Department of Mathematics, COMSATS University, Chak Shahzad, Islamabad, 44000, Pakistan.

${ }^{2}$ Department of Mathematics, Faculty of Physical Sciences, Ahmadu Bello University, Nigeria.

a e-mail:naimat0347@gmail.com

${ }^{b}$ e-mail: ssmohammed@abu.edu.ng

c e-mail:akbarazam@yahoo.com . 
(see, for instance, $[8,7,12,22]$ ). Moreover, all the generalizations of Banach fixed point theorem is further classified in two directions-either the contractive condition is replaced with a more generalized one or the axioms characterizing the ground set is enlarged or weakened. In the second case, some of these metric-like spaces are called semimetric, quasimetric, pseuodometric, $b$-metric, $K$-metric, to mention but a few. Along this line, by replacing the set of real numbers as the usual co-domain of a metric, Huang and Zhang [18] established the concept of cone metric as a generalization of metric spaces, thereby, establishing some fixed point theorems for contractive mappings on cone metric spaces. Starting from the year 2007, many authors have come up with various significant fixed point results in the frame of cone metric spaces (see, for example, $[19,26])$. The interested researcher may also want to go deeply into a comprehensive new survey on cone metric spaces by Aleksić et al. [4]

It is well-known that fixed point results involving rational contractive conditions cannot be extended or even meaningless in cone metric spaces. To tame this restriction, Azam et al. [5] initiated the concept of complex valued metric spaces and established sufficient conditions for the existence of common fixed points of a pair of mappings satisfying contractive type conditions involving rational expressions. Thereafter, the study of fixed point theorems concerning rational inequalities in complex valued metric spaces have been growing vigorously (see, for example, $[1,2,6,7,14,15,21])$. Along the line, the idea of $b$-metric space was presented by Bakhtin [9] in 1989. Rao [25] introduced the notion of fixed point results on complex valued $b$-metric spaces, which is broader than complex valued metric spaces. However, every complex valued $b$-metric space is a cone $b$-metric space over Banach algebra $\mathbb{C}$ in which the cone is normal with the coefficient of normality $K=1$, and where the cone has non-empty interior (that is, solid cone). Following [25], various authors have demonstrated fixed point results for different mappings fulfilling rational inequalities with regards to complex valued $b$-metric spaces (see, for instance, $[3,8])$.

Our contribution in this paper is to generalize the idea of extended $b$-metric spaces to complex valued extended $b$-metric spaces. In what follows hereafter, we recall some basic concepts that are necessary in the establishment of our main results. Most of these preliminaries are recorded from $[3,5,11,20]$.

Definition 1.1. Let $\mathbb{C}$ be the set of all complex numbers and $l_{1}, l_{2} \in \mathbb{C}$. The partial order on $\mathbb{C}$ is defined as:

$l_{1} \preceq l_{2}$, if and only if $\operatorname{Re}\left(l_{1}\right) \leq \operatorname{Re}\left(l_{2}\right)$ and $\operatorname{Im}\left(l_{1}\right) \leq \operatorname{Im}\left(l_{2}\right)$. This implies that $l_{1} \preceq l_{2}$ if one of the below conditions is fulfilled:

(i) $\operatorname{Re}\left(l_{1}\right)=\operatorname{Re}\left(l_{2}\right), \operatorname{Im}\left(l_{1}\right)<\operatorname{Im}\left(l_{2}\right)$,

(ii) $\operatorname{Re}\left(l_{1}\right)<\operatorname{Re}\left(l_{2}\right), \operatorname{Im}\left(l_{1}\right)=\operatorname{Im}\left(l_{2}\right)$,

(iii) $\operatorname{Re}\left(l_{1}\right)<\operatorname{Re}\left(l_{2}\right), \operatorname{Im}\left(l_{1}\right)<\operatorname{Im}\left(l_{2}\right)$,

(iv) $\operatorname{Re}\left(l_{1}\right)=\operatorname{Re}\left(l_{2}\right), \operatorname{Im}\left(l_{1}\right)=\operatorname{Im}\left(l_{2}\right)$.

Definition 1.2. Let $\mathbb{G}$ be a non-empty set. If the mapping $d: \mathbb{G} \times \mathbb{G} \rightarrow \mathbb{C}$ satisfies the following conditions:

(i) $0 \preceq d(l, m)$ and $d(l, m)=0 \Longleftrightarrow l=m$;

(ii) $d(l, m)=d(m, l)$;

(iii) $d(l, m) \preceq d(l, n)+d(n, m), \forall l, n, m \in \mathbb{G}$,

then $d$ is known as a complex valued (C.V.) metric on $G$, and the pair $(G, d)$ is said to be a C.V metric space.

Example 1.1. Let $X=[0,1]$ and $l, m \in \mathbb{G}$. Define $d: \mathbb{G} \times \mathbb{G} \rightarrow \mathbb{C}$ by

$$
d(l, m)= \begin{cases}0, & \text { if } \quad l=m \\ \frac{i}{2}, & \text { if } \quad l \neq m .\end{cases}
$$

Then $d$ is a C.V metric and hence $(\mathbb{G}, d)$ is a complex valued metric space.

Definition 1.3. Let $(G, d)$ be a C.V metric space.

(i) We say that a point $l \in \mathbb{G}$ is an interior point of a set $M \subseteq \mathbb{G}$, whenever there exists $0 \prec r \in \mathbb{C}$ such that

$$
B(l, r)=\{m \in \mathbb{G}: d(m, l) \prec r\},
$$

where $B(l, r)$ is an open ball with radius $r$, centered at $l$.

(ii) We say that a point $l \in \mathbb{G}$ is the limit point of a set $M \subseteq \mathbb{G}$ whenever for every $0 \prec r \in \mathbb{C}$, we have

$$
B(l, r) \bigcap M-\{l\} \neq \varnothing .
$$


Lemma 1.1. [3] Let $(G, d)$ be a C.V metric space and $\left\{l_{p}\right\}$ be a sequence in $\mathbb{G}$. Then $\left\{l_{p}\right\}$ is a convergent sequence if and only if $\left|d\left(l_{p}, l\right)\right| \rightarrow 0$ as $p \rightarrow \infty$.

Lemma 1.2. [3] Let $(G, d)$ be a C.V metric space and $\left\{l_{p}\right\}$ be a sequence in $G$. Then $\left\{l_{p}\right\}$ is a Cauchy sequence if and only if $\left|d\left(l_{p}, l_{q}\right)\right| \rightarrow 0$ as $p, q \rightarrow \infty$.

Definition 1.4. Let $\left(\mathbb{G}, d_{c}\right)$ be a $C . V$ metric space. We denote

$$
s(u)=\{z \in \mathbb{C}: u \preceq z\},
$$

for $l \in \mathbb{G}$ and $\mathbb{N} \in C B(\mathbb{G})$

$$
s(l, \mathbb{N})=\bigcup_{n \in \mathbb{N}} s\left(d_{c}(l, n)\right)=\bigcup_{n \in \mathbb{N}}\left\{z \in \mathbb{C}: d_{c}(l, n) \preceq z\right\},
$$

for $\mathbb{M}, \mathbb{N} \in C B(\mathbb{G})$, we have

$$
s(\mathbb{M}, \mathbb{N})=\left(\bigcap_{n \in \mathbb{N}} s(n, \mathbb{M})\right) \cap\left(\bigcap_{m \in \mathbb{M}} s(m, \mathbb{N})\right) .
$$

Definition 1.5. Let $(G, d)$ be a C.V metric space.

(i) Let $\mathbb{T}: \mathbb{G} \rightarrow C B(\mathbb{G})$ be a multi-valued mapping. For $l \in \mathbb{G}$ and $M \in C B(G)$, define

$$
W_{l}(M)=\{d(l, a): a \in M\},
$$

and for $l, m \in \mathbb{G}$, and $\mathbb{T} m \in C B(\mathbb{G})$, we have

$$
W_{l}(\mathbb{T} m)=\{d(l, u): u \in \mathbb{T} m\} .
$$

(ii) A mapping $F: G \rightarrow 2^{\mathbb{C}}$ is said to bounded below if for each $l \in \mathbb{G}$ there exists $w \in \mathbb{C}$ such that $z_{l} \preceq w$ for all $w \in F_{l}$.

(iii) For multi-valued mapping $J: G \rightarrow C B(G)$, we say that it has lower bound property on $\left(\mathbb{G}, d_{c}\right)$ if for any $l \in \mathbb{G}$ the mapping $F_{l}: G \rightarrow 2^{\mathrm{C}}$ defined by $F_{l}(\mathrm{Jv})=W_{l}(F v)$ is bounded below. This means that for $l, v \in \mathbb{G}$ there is an element $l_{l}(J v) \in \mathbb{C}$ such that $l_{l}(J v) \preceq$ a for all $a \in W_{l}(J v)$, where $l_{l}(J v)$ is said to be a lower bound of $J$ corresponding to $(l, v)$.

(iv) For multi-valued mapping $J: \mathbb{G} \rightarrow C B(\mathbb{G})$, we say that it has greatest lower bound property (g.l.b property) on $\left(\mathbb{G}, d_{c}\right)$ if the g.l.b of $W_{l}(J v)$ exists in $\mathbb{C}$ for all $l, v \in \mathbb{G}$. We denote the g.l.b of $W_{l}(J v)$ by $d_{c}(l, J v)$ and define it as:

$$
d_{c}(l, J v)=\inf \left\{d_{c}(l, a): a \in J v\right\} .
$$

Definition 1.6. Let $\left(\mathbb{G}, d_{c}\right)$ be a $C . V$ metric space and $S, \mathbb{T}: G \rightarrow C B(G)$ be multi-valued mappings.

(i) A point $l \in \mathbb{G}$ is called a fixed point of $\mathbb{T}$ if $l \in \mathbb{T} l$.

(ii) A point $l \in \mathbb{G}$ is called a common fixed point of $\mathrm{S}$ and $\mathbb{T}$ if $l \in \mathrm{S} l$ and $l \in \mathbb{T} l$.

Definition 1.7. [9] Let $\mathbb{G}$ be a non-empty set and $\tau \geq 1$ be a real number. A function $d_{c}: \mathbb{G} \times \mathbb{G} \rightarrow \mathbb{C}$ is called C.V $b$-metric space, if for all $l, m, n \in \mathbb{G}$, the following conditions hold.

(i) $0 \preceq d_{c}(l, m)$ and $d_{c}(l, m)=0$ if and only if $l=m$;

(ii) $d_{c}(l, m)=d_{c}(m, l)$;

(iii) $d_{c}(l, m) \preceq \tau\left[d_{c}(l, n)+d_{c}(n, m)\right]$.

Then $d_{c}$ is called a C.V b-metric on $\mathbb{G}$ and the pair $\left(\mathbb{G}, d_{c}\right)$ is called a C.V b-metric space.

Example 1.2. [30] Let $\mathbb{G}=[0,1]$. Define a mapping $d_{c}: \mathbb{G} \times \mathbb{G} \rightarrow \mathbb{C}$ by

$$
d_{c}(l, m)=|l-m|^{2}+i|l-m|^{2}
$$

for all $l, m \in \mathbb{G}$. Then $\left(\mathbb{G}, d_{c}\right)$ is a $C . V$ b-metric space with $\tau=2$.

Definition 1.8. Let $\mathbb{G}$ be a non-empty set and $\theta: \mathbb{G} \times \mathbb{G} \rightarrow[1, \infty)$ be a function. Then $d_{\theta}: \mathbb{G} \times \mathbb{G} \rightarrow \mathbb{C}$ is known as a C.V extended $b$-metric space if the following are satisfied for all $l, m, n \in \mathbb{G}$ :

(i) $0 \preceq d_{\theta}(l, m)$ and $d_{\theta}(l, m)=0$ if and only if $l=m$;

(ii) $d_{\theta}(l, m)=d_{\theta}(m, l)$;

(iii) $d_{\theta}(l, n) \preceq \theta(l, n)\left[d_{\theta}(l, m)+d_{\theta}(m, n)\right]$.

then the pair $\left(\mathbb{G}, d_{\theta}\right)$ is known as a $C . V$ extended b-matric space. 
Example 1.3. [10] Let $\mathbb{G}$ be a non-empty set and $\theta: \mathbb{G} \times \mathbb{G} \rightarrow[1, \infty)$ be defined as:

$$
\theta(l, m)=\frac{1+l+m}{l+m}
$$

Further, Let

(i) $d_{\theta}(l, m)=\frac{i}{l m}$, for all $l, m \in(0,1]$;

(ii) $d_{\theta}(l, m)=0 \Leftrightarrow l=m$ for all $l, m \in[0,1]$;

(iii) $d_{\theta}(l, 0)=d_{\theta}(0, l)=\frac{i}{l}$ for all $l \in(0,1]$.

Then the pair $\left(G, d_{\theta}\right)$ is known as a $C . V$ extended b-metric space.

Example 1.4. Let $\mathbb{G}=[0, \infty) . \theta: \mathbb{G} \times \mathbb{G} \rightarrow[1, \infty)$ be a function defined by $\theta(l, m)=1+l+m$ and $d_{\theta}: \mathbb{G} \times \mathbb{G} \longrightarrow \mathbb{C}$ be given as

$$
d_{\theta}(l, m)= \begin{cases}0, & \text { if } l=m \\ i, & \text { if } l \neq m\end{cases}
$$

Then $\left(\mathbb{G}, d_{\theta}\right)$ is known as a C.V extended b-metric space.

In what follows hereafter, we present our main results.

\section{Fixed point results for almost contraction in complex valued extended $b$-metric spaces}

We start this section with the following definition.

Definition 2.1. Let $\left(\mathbb{G}, d_{\theta}\right)$ be a complex valued extended b-metric space. A mapping $\mathbb{T}: \mathbb{G} \rightarrow \mathbb{G}$ is said to be an almost contraction if there exists $r \in(0,1)$ and $\mathbb{L} \geq 0$ such that for all $l, m \in \mathbb{G}$,

$$
d_{\theta}(\mathbb{T} l, \mathbb{T} m) \leq r d_{\theta}(l, m)+\mathbb{L} d_{\theta}(m, \mathbb{T} l) .
$$

Theorem 2.1. Let $\left(\mathbb{G}, d_{\theta}\right)$ be a complete complex valued extended b-metric space; let $\theta: \mathbb{G} \times \mathbb{G} \rightarrow[1, \infty)$ and $\mathbb{S}, \mathbb{T}:$ $\mathbb{G} \times \mathbb{G} \rightarrow C B(\mathbb{G})$ be a pair of multi-valued mappings with g.l.b property such that

$$
\begin{aligned}
& \lambda_{1} d_{\theta}(l, m)+\lambda_{2}\left[d_{\theta}(l, \mathbb{S} l)+d_{\theta}(m, \mathbb{T} m)\right]+\lambda_{3}\left[d_{\theta}(m, \mathbb{S} l)+d_{\theta}(l, \mathbb{T} m)\right] \\
& +\lambda_{4} \frac{d_{\theta}(m, \mathbb{T} m)\left[1+d_{\theta}(l, \mathbb{S} l)\right]}{1+d_{\theta}(l, m)}+\lambda_{5} \frac{d_{\theta}(m, \mathbb{S} l)\left[1+d_{\theta}(l, \mathbb{T} m)\right]}{1+d_{\theta}(l, m)} \\
& +\lambda_{6} \frac{d_{\theta}(l, m)\left[1+d_{\theta}(l, \mathbb{S} l)+d_{\theta}(m, \mathbb{S} l)\right]}{1+d_{\theta}(l, m)}+\mathbb{L} d_{\theta}(m, \mathbb{S} l) \in s(\mathbb{S} l, \mathbb{T} m)
\end{aligned}
$$

for all $l, m \in \mathbb{G}$ and $\lambda_{1}, \lambda_{2}, \lambda_{3}, \lambda_{4}, \lambda_{5}, \lambda_{6}, \mathbb{L}$ are nonnegative real numbers with $\lambda_{1}+2 \lambda_{2}$ $+2 \lambda_{3} \theta\left(l_{0}, l_{2}\right)+\lambda_{4}+\lambda_{6}<1, k\left(1-\lambda_{2}-\lambda_{3} \theta\left(l_{0}, l_{2}\right)-\lambda_{4}\right)=\left(\lambda_{1}+\lambda_{2}+\lambda_{3} \theta\left(l_{0}, l_{2}\right)+\lambda_{6}\right)$ where $k \in[0, \infty)$ be such that for each $l_{o} \in \mathbb{G}, \lim _{p, q \rightarrow \infty} \theta\left(l_{p}, l_{q}\right)<\frac{1}{k}$. Then $\mathbb{S}$ and $\mathbb{T}$ have a common fixed point.

Proof. Let $l_{o} \in \mathbb{G}$, then $\mathbb{T} l_{o}$ is non empty, so we take $l_{1} \in \mathrm{S} l_{0}$. Thus, from (2.1), we have

$$
\begin{aligned}
& \lambda_{1} d_{\theta}\left(l_{o}, l_{1}\right)+\lambda_{2}\left[d_{\theta}\left(l_{o}, S l_{o}\right)+d_{\theta}\left(l_{1}, \mathbb{T} l_{1}\right)\right]+\lambda_{3}\left[d_{\theta}\left(l_{1}, S l_{o}\right)+d_{\theta}\left(l_{o}, \mathbb{T} l_{1}\right)\right] \\
& +\lambda_{4} \frac{d_{\theta}\left(l_{1}, \mathbb{T} l_{1}\right)\left[1+d_{\theta}\left(l_{o}, S l_{o}\right)\right]}{1+d_{\theta}\left(l_{o}, l_{1}\right)}+\lambda_{5} \frac{d_{\theta}\left(l_{1}, S l_{o}\right)\left[1+d_{\theta}\left(l_{o}, \mathbb{T} l_{1}\right)\right]}{1+d_{\theta}\left(l_{o}, l_{1}\right)} \\
& +\lambda_{6} \frac{d_{\theta}\left(l_{o}, l_{1}\right)\left[1+d_{\theta}\left(l_{o}, S l_{o}\right)+d_{\theta}\left(l_{1}, S l_{o}\right)\right]}{1+d_{\theta}\left(l_{o}, l_{1}\right)}+\mathbb{L} d_{\theta}\left(l_{1}, S l_{o}\right) \in s\left(S l_{o}, \mathbb{T} l_{1}\right) \\
& \lambda_{1} d_{\theta}\left(l_{o}, l_{1}\right)+\lambda_{2}\left[d_{\theta}\left(l_{o}, S l_{o}\right)+d_{\theta}\left(l_{1}, T l_{1}\right)\right]+\lambda_{3}\left[d_{\theta}\left(l_{1}, S l_{o}\right)+d_{\theta}\left(l_{o}, \mathbb{T} l_{1}\right)\right] \\
& +\lambda_{4} \frac{d_{\theta}\left(l_{1}, \mathbb{T} l_{1}\right)\left[1+d_{\theta}\left(l_{o}, S l_{o}\right)\right]}{1+d_{\theta}\left(l_{o}, l_{1}\right)}+\lambda_{5} \frac{d_{\theta}\left(l_{1}, S l_{o}\right)\left[1+d_{\theta}\left(l_{o}, \mathbb{T} l_{1}\right)\right]}{1+d_{\theta}\left(l_{o}, l_{1}\right)}
\end{aligned}
$$




$$
+\lambda_{6} \frac{d_{\theta}\left(l_{o}, l_{1}\right)\left[1+d_{\theta}\left(l_{o}, \mathbb{S} l_{o}\right)+d_{\theta}\left(l_{1}, S l_{o}\right)\right]}{1+d_{\theta}\left(l_{o}, l_{1}\right)}+\mathbb{L} d_{\theta}\left(l_{1}, S l_{o}\right) \in \bigcap_{a \in \mathbb{S} l_{o}} s\left(a, \mathbb{T} l_{1}\right)
$$

This gives

$$
\begin{aligned}
& \lambda_{1} d_{\theta}\left(l_{o}, l_{1}\right)+\lambda_{2}\left[d_{\theta}\left(l_{o}, \mathrm{~S} l_{o}\right)+d_{\theta}\left(l_{1}, \mathbb{T} l_{1}\right)\right]+\lambda_{3}\left[d_{\theta}\left(l_{1}, \mathrm{~S} l_{o}\right)+d_{\theta}\left(l_{o}, \mathbb{T} l_{1}\right)\right] \\
& +\lambda_{4} \frac{d_{\theta}\left(l_{1}, \mathbb{T} l_{1}\right)\left[1+d_{\theta}\left(l_{o}, \mathrm{~S} l_{o}\right)\right]}{1+d_{\theta}\left(l_{0}, l_{1}\right)}+\lambda_{5} \frac{d_{\theta}\left(l_{1}, \mathrm{~S} l_{o}\right)\left[1+d_{\theta}\left(l_{o}, \mathbb{T} l_{1}\right)\right]}{1+d_{\theta}\left(l_{o}, l_{1}\right)} \\
& +\lambda_{6} \frac{d_{\theta}\left(l_{o}, l_{1}\right)\left[1+d_{\theta}\left(l_{o}, S l_{o}\right)+d_{\theta}\left(l_{1}, S l_{o}\right)\right]}{1+d_{\theta}\left(l_{o}, l_{1}\right)}+\mathbb{L} d_{\theta}\left(l_{1}, S l_{o}\right) \in s\left(a, \mathbb{T} l_{1}\right), \quad \forall a \in \mathbb{S} l_{o} .
\end{aligned}
$$

Since $l_{1} \in \mathbb{S} l_{0}$, then

$$
\begin{aligned}
& \lambda_{1} d_{\theta}\left(l_{o}, l_{1}\right)+\lambda_{2}\left[d_{\theta}\left(l_{o}, \mathbb{S} l_{o}\right)+d_{\theta}\left(l_{1}, \mathbb{T} l_{1}\right)\right]+\lambda_{3}\left[d_{\theta}\left(l_{1}, S l_{o}\right)+d_{\theta}\left(l_{o}, \mathbb{T} l_{1}\right)\right] \\
& +\lambda_{4} \frac{d_{\theta}\left(l_{1}, \mathbb{T} l_{1}\right)\left[1+d_{\theta}\left(l_{o}, S l_{o}\right)\right]}{1+d_{\theta}\left(l_{o}, l_{1}\right)}+\lambda_{5} \frac{d_{\theta}\left(l_{1}, \mathbb{S} l_{o}\right)\left[1+d_{\theta}\left(l_{o}, \mathbb{T} l_{1}\right)\right]}{1+d_{\theta}\left(l_{o}, l_{1}\right)} \\
& +\lambda_{6} \frac{d_{\theta}\left(l_{o}, l_{1}\right)\left[1+d_{\theta}\left(l_{0}, S l_{o}\right)+d_{\theta}\left(l_{1}, S l_{o}\right)\right]}{1+d_{\theta}\left(l_{o}, l_{1}\right)}+\mathbb{L} d_{\theta}\left(l_{1}, S l_{o}\right) \in s\left(l_{1}, \mathbb{T} l_{1}\right) \\
& \lambda_{1} d_{\theta}\left(l_{o}, l_{1}\right)+\lambda_{2}\left[d_{\theta}\left(l_{o}, S l_{o}\right)+d_{\theta}\left(l_{1}, \mathbb{T} l_{1}\right)\right]+\lambda_{3}\left[d_{\theta}\left(l_{1}, S l_{o}\right)+d_{\theta}\left(l_{o}, \mathbb{T} l_{1}\right)\right] \\
& +\lambda_{4} \frac{d_{\theta}\left(l_{1}, \mathbb{T} l_{1}\right)\left[1+d_{\theta}\left(l_{o}, S l_{o}\right)\right]}{1+d_{\theta}\left(l_{o}, l_{1}\right)}+\lambda_{5} \frac{d_{\theta}\left(l_{1}, S l_{o}\right)\left[1+d_{\theta}\left(l_{o}, \mathbb{T} l_{1}\right)\right]}{1+d_{\theta}\left(l_{o}, l_{1}\right)} \\
& +\lambda_{6} \frac{d_{\theta}\left(l_{o}, l_{1}\right)\left[1+d_{\theta}\left(l_{o}, S l_{o}\right)+d_{\theta}\left(l_{1}, S l_{o}\right)\right]}{1+d_{\theta}\left(l_{o}, l_{1}\right)}+\mathbb{L} d_{\theta}\left(l_{1}, S l_{o}\right) \in \bigcup_{b \in \mathbb{T} l_{1}} s\left(l_{1}, b\right) .
\end{aligned}
$$

Therefore, there exists $l_{2} \in \mathbb{T} l_{1}$ such that

$$
\begin{aligned}
& \lambda_{1} d_{\theta}\left(l_{o}, l_{1}\right)+\lambda_{2}\left[d_{\theta}\left(l_{o}, \mathbb{S} l_{o}\right)+d_{\theta}\left(l_{1}, \mathbb{T} l_{1}\right)\right]+\lambda_{3}\left[d_{\theta}\left(l_{1}, S l_{o}\right)+d_{\theta}\left(l_{o}, \mathbb{T} l_{1}\right)\right] \\
& +\lambda_{4} \frac{d_{\theta}\left(l_{1}, \mathbb{T} l_{1}\right)\left[1+d_{\theta}\left(l_{o}, S l_{o}\right)\right]}{1+d_{\theta}\left(l_{o}, l_{1}\right)}+\lambda_{5} \frac{d_{\theta}\left(l_{1}, S l_{o}\right)\left[1+d_{\theta}\left(l_{o}, \mathbb{T} l_{1}\right)\right]}{1+d_{\theta}\left(l_{o}, l_{1}\right)} \\
& +\lambda_{6} \frac{d_{\theta}\left(l_{o}, l_{1}\right)\left[1+d_{\theta}\left(l_{o}, S l_{o}\right)+d_{\theta}\left(l_{1}, S l_{o}\right)\right]}{1+d_{\theta}\left(l_{o}, l_{1}\right)}+\mathbb{L} d_{\theta}\left(l_{1}, S l_{o}\right) \in s\left(d_{\theta}\left(l_{1}, l_{2}\right)\right) .
\end{aligned}
$$

By definition and the g.l.b property of $\mathrm{S}$ and $\mathbb{T}$, we have

$$
\begin{aligned}
d_{\theta}\left(l_{1}, l_{2}\right) & \preceq \lambda_{1} d_{\theta}\left(l_{o}, l_{1}\right)+\lambda_{2}\left[d_{\theta}\left(l_{o}, S l_{o}\right)+d_{\theta}\left(l_{1}, \mathbb{T} l_{1}\right)\right]+\lambda_{3}\left[d_{\theta}\left(l_{1}, \mathbb{S} l_{o}\right)+d_{\theta}\left(l_{o}, \mathbb{T} l_{1}\right)\right] \\
& +\lambda_{4} \frac{d_{\theta}\left(l_{1}, \mathbb{T} l_{1}\right)\left[1+d_{\theta}\left(l_{o}, S l_{o}\right)\right]}{1+d_{\theta}\left(l_{o}, l_{1}\right)}+\lambda_{5} \frac{d_{\theta}\left(l_{1}, S l_{o}\right)\left[1+d_{\theta}\left(l_{o}, \mathbb{T} l_{1}\right)\right]}{1+d_{\theta}\left(l_{o}, l_{1}\right)} \\
& +\lambda_{6} \frac{d_{\theta}\left(l_{o}, l_{1}\right)\left[1+d_{\theta}\left(l_{o}, S l_{o}\right)+d_{\theta}\left(l_{1}, S l_{o}\right)\right]}{1+d_{\theta}\left(l_{o}, l_{1}\right)}+\mathbb{L}, d_{\theta}\left(l_{1}, S l_{o}\right)
\end{aligned}
$$

from which we have

$$
\begin{aligned}
d_{\theta}\left(l_{1}, l_{2}\right) & \preceq \lambda_{1} d_{\theta}\left(l_{o}, l_{1}\right)+\lambda_{2}\left[d_{\theta}\left(l_{o}, l_{1}\right)+d_{\theta}\left(l_{1}, l_{2}\right)\right]+\lambda_{3}\left[d_{\theta}\left(l_{1}, l_{1}\right)+d_{\theta}\left(l_{o}, l_{2}\right)\right] \\
& +\lambda_{4} \frac{d_{\theta}\left(l_{1}, l_{2}\right)\left[1+d_{\theta}\left(l_{o}, l_{1}\right)\right]}{1+d_{\theta}\left(l_{o}, l_{1}\right)}+\lambda_{5} \frac{d_{\theta}\left(l_{1}, l_{1}\right)\left[1+d_{\theta}\left(l_{o}, l_{2}\right)\right]}{1+d_{\theta}\left(l_{o}, l_{1}\right)} \\
& +\lambda_{6} \frac{d_{\theta}\left(l_{o}, l_{1}\right)\left[1+d_{\theta}\left(l_{o}, l_{1}\right)+d_{\theta}\left(l_{1}, l_{1}\right)\right]}{1+d_{\theta}\left(l_{0}, l_{1}\right)}+\mathbb{L} d_{\theta}\left(l_{1}, l_{1}\right) .
\end{aligned}
$$

This implies

$$
\begin{aligned}
\left|d_{\theta}\left(l_{1}, l_{2}\right)\right| & \leq \lambda_{1}\left|d_{\theta}\left(l_{o}, l_{1}\right)\right|+\lambda_{2}\left[\left|d_{\theta}\left(l_{o}, l_{1}\right)\right|+\left|d_{\theta}\left(l_{1}, l_{2}\right)\right|\right] \\
& +\lambda_{3}\left|d_{\theta}\left(l_{0}, l_{2}\right)\right|+\lambda_{4}\left|d_{\theta}\left(l_{1}, l_{2}\right)\right|+\lambda_{6}\left|d_{\theta}\right|\left(l_{o}, l_{1}\right) \mid .
\end{aligned}
$$


Since $\left|d_{\theta}\left(l_{1}, l_{2}\right)\right|<1+\left|d_{\theta}\left(l_{1}, l_{2}\right)\right|$,

$$
\left|d_{\theta}\left(l_{1}, l_{2}\right)\right| \leq \frac{\left(\lambda_{1}+\lambda_{2}+\lambda_{3} \theta\left(l_{o}, l_{2}\right)+\lambda_{6}\right)}{\left(1-\lambda_{2}-\lambda_{3} \theta\left(l_{o}, l_{2}\right)-\lambda_{4}\right)}\left|d_{\theta}\left(l_{0}, l_{1}\right)\right| .
$$

Then, we get

$$
\left|d_{\theta}\left(l_{1}, l_{2}\right)\right| \leq k\left|d_{\theta}\left(l_{0}, l_{1}\right)\right|
$$

Inductively, we can find a sequence $\left\{l_{p}\right\}$ in $\mathbb{G}$ such that

$$
\begin{aligned}
\left|d_{\theta}\left(l_{1}, l_{2}\right)\right| & \leq \kappa\left|d_{\theta}\left(l_{0}, l_{1}\right)\right| \\
\left|d_{\theta}\left(l_{2}, l_{3}\right)\right| & \leq \kappa^{2}\left|d_{\theta}\left(l_{0}, l_{1}\right)\right| \\
\cdot & \cdot \\
\cdot & \cdot \\
\left|d_{\theta}\left(l_{p}, l_{p+1}\right)\right| & \leq \kappa^{p}\left|d_{\theta}\left(l_{0}, l_{1}\right)\right| .
\end{aligned}
$$

Now, by triangular inequality, for $q>p$, we have

$$
\begin{aligned}
d_{\theta}\left(l_{p}, l_{q}\right) & \preceq \theta\left(l_{p}, l_{q}\right) k^{p} d_{\theta}\left(l_{o}, l_{1}\right)+\theta\left(l_{p}, l_{q}\right) \theta\left(l_{p+1}, l_{q}\right) k^{p+1} d_{\theta}\left(l_{o}, l_{1}\right)+\cdots \\
& +\theta\left(l_{p}, l_{q}\right) \theta\left(l_{p+1}, l_{q}\right) \cdots \theta\left(l_{q-2}, l_{q}\right) \theta\left(l_{q-1}, l_{q}\right) k^{q-1} d_{\theta}\left(l_{o}, l_{1}\right) \\
d_{\theta}\left(l_{p}, l_{q}\right) & \preceq d_{\theta}\left(l_{0}, l_{1}\right)\left[\theta\left(l_{p}, l_{q}\right) k^{p}+\theta\left(l_{p}, l_{q}\right) \theta\left(l_{p+1}, l_{q}\right) k^{p+1}+\cdots\right. \\
& \left.+\theta\left(l_{p}, l_{q}\right) \theta\left(l_{p+1}, l_{q}\right) \ldots \theta\left(l_{q-2}, l_{q}\right) \theta\left(l_{q-1}, l_{p}\right) k^{q-1}\right]
\end{aligned}
$$

Since $\lim _{p, q \rightarrow \infty} \theta\left(l_{p}, l_{q}\right) k<1$, so the series $\sum_{p=1}^{\infty} k^{p} \prod_{i=1}^{p} \theta\left(l_{i}, l_{q}\right)$ converges by ratio test for each $q \in \mathbb{N}$. Let

$$
S=\sum_{p=1}^{\infty} k^{p} \prod_{i=1}^{p} \theta\left(l_{i}, l_{q}\right), S_{p}=\sum_{j=1}^{p} k^{j} \prod_{i=1}^{p} \theta\left(l_{i}, l_{q}\right) .
$$

Thus, for $q>p$, the above can be written as

$$
d_{\theta}\left(l_{p}, l_{q}\right) \preceq d_{\theta}\left(l_{0}, l_{1}\right)\left[S_{q-1}-S_{P}\right]
$$

and

$$
\left|d_{\theta}\left(l_{p}, l_{q}\right)\right| \leq\left|d_{\theta}\left(l_{0}, l_{1}\right)\right|\left[S_{q-1}-S_{p}\right]
$$

Now, by taking $p \rightarrow \infty$, we get

$$
\left|d_{\theta}\left(l_{p}, l_{q}\right)\right| \rightarrow 0
$$

By lemma (2.4), $\left\{l_{p}\right\}$ is a Cauchy sequence in $G$. By completeness of $\mathbb{G}$, there exists some $r \in \mathbb{G}$ such that

$$
\lim _{p \rightarrow \infty} l_{p}=r .
$$

Now, we show that $r \in \mathrm{S} r$ and $r \in \mathbb{T} r$. From (2.1), we have

$$
\begin{aligned}
& \lambda_{1} d_{\theta}\left(l_{2 p}, r\right)+\lambda_{2}\left[d_{\theta}\left(l_{2 p}, \mathrm{~S} l_{2 p}\right)+d_{\theta}(r, \mathbb{T} r)\right]+\lambda_{3}\left[d_{\theta}\left(r, S l_{2 p}\right)+d_{\theta}\left(l_{2 p}, \mathbb{T} r\right)\right] \\
& +\lambda_{4} \frac{d_{\theta}(r, \mathbb{T} r)\left[1+d_{\theta}\left(l_{2 p}, S l_{2 p}\right)\right]}{1+d_{\theta}\left(l_{2 p}, r\right)}+\lambda_{5} \frac{d_{\theta}\left(r, S l_{2 p}\right)\left[1+d_{\theta}\left(l_{2 p}, \mathbb{T} r\right)\right]}{1+d_{\theta}\left(l_{2 p}, r\right)} \\
& +\lambda_{6} \frac{d_{\theta}\left(l_{2 p}, r\right)\left[1+d_{\theta}\left(l_{2 p}, S l_{2 p}\right)+d_{\theta}\left(r, S l_{2 p}\right)\right]}{1+d_{\theta}\left(l_{2 p}, r\right)}+\mathbb{L} d_{\theta}\left(r, S l_{2 p}\right) \in s\left(\mathbb{S} l_{2 p}, \mathbb{T} r\right) .
\end{aligned}
$$


This implies

$$
\begin{aligned}
& \lambda_{1} d_{\theta}\left(l_{2 p}, r\right)+\lambda_{2}\left[d_{\theta}\left(l_{2 p}, S_{2 p}\right)+d_{\theta}(r, \mathbb{T} r)\right]+\lambda_{3}\left[d_{\theta}\left(r, S l_{2 p}\right)+d_{\theta}\left(l_{2 p}, \mathbb{T} r\right)\right] \\
& +\lambda_{4} \frac{d_{\theta}(r, \mathbb{T} r)\left[1+d_{\theta}\left(l_{2 p}, \mathbb{S} l_{2 p}\right)\right]}{1+d_{\theta}\left(l_{2 p}, r\right)}+\lambda_{5} \frac{d_{\theta}\left(r, \mathbb{S} l_{2 p}\right)\left[1+d_{\theta}\left(l_{2 p}, \mathbb{T} r\right)\right]}{1+d_{\theta}\left(l_{2 p}, r\right)} \\
& +\lambda_{6} \frac{d_{\theta}\left(l_{2 p}, r\right)\left[1+d_{\theta}\left(l_{2 p}, S l_{2 p}\right)+d_{\theta}\left(r, S l_{2 p}\right)\right]}{1+d_{\theta}\left(l_{2 p}, r\right)}+\mathbb{L} d_{\theta}\left(r, S l_{2 p}\right) \in \bigcap_{a \in S l_{2 p}} s(a, \mathbb{T} r) . \\
& \lambda_{1} d_{\theta}\left(l_{2 p}, r\right)+\lambda_{2}\left[d_{\theta}\left(l_{2 p}, \mathbf{S} l_{2 p}\right)+d_{\theta}(r, \mathbb{T} r)\right]+\lambda_{3}\left[d_{\theta}\left(r, \mathbb{S} l_{2 p}\right)+d_{\theta}\left(l_{2 p}, \mathbb{T} r\right)\right] \\
& +\lambda_{4} \frac{d_{\theta}(r, \mathbb{T} r)\left[1+d_{\theta}\left(l_{2 p}, \mathbb{S} l_{2 p}\right)\right]}{1+d_{\theta}\left(l_{2 p}, r\right)}+\lambda_{5} \frac{d_{\theta}\left(r, S l_{2 p}\right)\left[1+d_{\theta}\left(l_{2 p}, \mathbb{T} r\right)\right]}{1+d_{\theta}\left(l_{2 p}, r\right)} \\
& +\lambda_{6} \frac{d_{\theta}\left(l_{2 p}, r\right)\left[1+d_{\theta}\left(l_{2 p}, \mathbb{S} l_{2 p}\right)+d_{\theta}\left(r, \mathbb{S} l_{2 p}\right)\right]}{1+d_{\theta}\left(l_{2 p}, r\right)}+\mathbb{L} d_{\theta}\left(r, S l_{2 p}\right) \in s(a, \mathbb{T} r), \quad \forall a \in \mathbb{S} l_{2 n} .
\end{aligned}
$$

Since $l_{2 p+1} \in \mathrm{S} l_{2 p}$, therefore

$$
\begin{aligned}
& \lambda_{1} d_{\theta}\left(l_{2 p}, r\right)+\lambda_{2}\left[d_{\theta}\left(l_{2 p}, \mathbb{S} l_{2 p}\right)+d_{\theta}(r, \mathbb{T} r)\right]+\lambda_{3}\left[d_{\theta}\left(r, \mathbb{S} l_{2 p}\right)+d_{\theta}\left(l_{2 p}, \mathbb{T} r\right)\right] \\
&+ \lambda_{4} \frac{d_{\theta}(r, \mathbb{T} r)\left[1+d_{\theta}\left(l_{2 p}, S l_{2 p}\right)\right]}{1+d_{\theta}\left(l_{2 p}, r\right)}+\lambda_{5} \frac{d_{\theta}\left(r, \mathbb{S} l_{2 p}\right)\left[1+d_{\theta}\left(l_{2 p}, \mathbb{T} r\right)\right]}{1+d_{\theta}\left(l_{2 p}, r\right)} \\
&+ \lambda_{6} \frac{d_{\theta}\left(l_{2 p}, r\right)\left[1+d_{\theta}\left(l_{2 p}, S l_{2} p\right)+d_{\theta}\left(r, \mathbb{S} l_{2 p}\right)\right]}{1+d_{\theta}\left(l_{2 p}, r\right)}+\mathbb{L} d_{\theta}\left(r, S l_{2 p}\right) \in s\left(l_{2 p+1}, \mathbb{T} r\right), \\
& \lambda_{1} d_{\theta}\left(l_{2 p}, r\right)+\lambda_{2}\left[d_{\theta}\left(l_{2 p}, S l_{2 p}\right)+d_{\theta}(r, \mathbb{T} r)\right]+\lambda_{3}\left[d_{\theta}\left(r, S l_{2 p}\right)+d_{\theta}\left(l_{2 p}, \mathbb{T} r\right)\right] \\
&+\lambda_{4} \frac{d_{\theta}(r, \mathbb{T} r)\left[1+d_{\theta}\left(l_{2 p}, \mathbb{S} l_{2 p}\right)\right]}{1+d_{\theta}\left(l_{2 p}, r\right)}+\lambda_{5} \frac{d_{\theta}\left(r, \mathbb{S} l_{2 p}\right)\left[1+d_{\theta}\left(l_{2 p}, \mathbb{T} r\right)\right]}{1+d_{\theta}\left(l_{2 p}, r\right)} \\
&+\lambda_{6} \frac{d_{\theta}\left(l_{2 p}, r\right)\left[1+d_{\theta}\left(l_{2 p}, \mathbb{S} l_{2} p\right)+d_{\theta}\left(r, \mathbb{S} l_{2 p}\right)\right]}{1+d_{\theta}\left(l_{2 p}, r\right)}+\mathbb{L} d_{\theta}\left(r, S l_{2 p}\right) \in \bigcup_{b \in \mathbb{T} r} s\left(d_{\theta}\left(l_{2 p+1}, b\right)\right) .
\end{aligned}
$$

This implies that there exists some $r_{p} \in \mathbb{T} r$ such that

$$
\begin{gathered}
\lambda_{1} d_{\theta}\left(l_{2 p}, r\right)+\lambda_{2}\left[d_{\theta}\left(l_{2 p}, \mathbb{S} l_{2 p}\right)+d_{\theta}(r, \mathbb{T} r)\right]+\lambda_{3}\left[d_{\theta}\left(r, \mathbb{S} l_{2 p}\right)+d_{\theta}\left(l_{2 p}, \mathbb{T} r\right)\right] \\
+\lambda_{4} \frac{d_{\theta}(r, \mathbb{T} r)\left[1+d_{\theta}\left(l_{2 p}, S l_{2 p}\right)\right]}{1+d_{\theta}\left(l_{2 p}, r\right)}+\lambda_{5} \frac{d_{\theta}\left(r, \mathbb{S} l_{2 p}\right)\left[1+d_{\theta}\left(l_{2 p}, \mathbb{T} r\right)\right]}{1+d_{\theta}\left(l_{2 p}, r\right)} \\
+\lambda_{6} \frac{d_{\theta}\left(l_{2 p}, r\right)\left[1+d_{\theta}\left(l_{2 p}, S l_{2 p}\right)+d_{\theta}\left(r, S l_{2 p}\right)\right]}{1+d_{\theta}\left(l_{2 p}, r\right)}+\mathbb{L} d_{\theta}\left(r, S l_{2 p}\right) \in s\left(d_{(}\left(l_{2 p+1}, r_{p}\right)\right), \\
d_{\theta}\left(l_{2 p+1}, r_{p}\right) \preceq \lambda_{1} d_{\theta}\left(l_{2 p}, r\right)+\lambda_{2}\left[d_{\theta}\left(l_{2 p}, \mathbb{S} l_{2 p}\right)+d_{\theta}(r, \mathbb{T} r)\right]+\lambda_{3}\left[d_{\theta}\left(r, \mathbb{S} l_{2 p}\right)+d_{\theta}\left(l_{2 p}, \mathbb{T} r\right)\right] \\
+\lambda_{4} \frac{d_{\theta}(r, \mathbb{T} r)\left[1+d_{\theta}\left(l_{2 p}, S l_{2 p}\right)\right]}{1+d_{\theta}\left(l_{2 p}, r\right)}+\lambda_{5} \frac{d_{\theta}\left(r, S l_{2 p}\right)\left[1+d_{\theta}\left(l_{2 p}, \mathbb{T} r\right)\right]}{1+d_{\theta}\left(l_{2 p}, r\right)} \\
+\lambda_{6} \frac{d_{\theta}\left(l_{2 p}, r\right)\left[1+d_{\theta}\left(l_{2 p}, S l_{2 p}\right)+d_{\theta}\left(r, \mathbb{S} l_{2 p}\right)\right]}{1+d_{\theta}\left(l_{2 p}, r\right)}+\mathbb{L} d_{\theta}\left(r, S l_{2 p}\right), \\
d_{\theta}\left(l_{2 p+1}, r_{p}\right) \preceq \lambda_{1} d_{\theta}\left(l_{2 p}, r\right)+\lambda_{2}\left[d_{\theta}\left(l_{2 p}, l_{2 p+1}\right)+d_{\theta}\left(r, r_{p}\right)\right]+\lambda_{3}\left[d_{\theta}\left(r, l_{2 p+1}\right)+d_{\theta}\left(l_{2 p}, r_{p}\right)\right] \\
+\lambda_{4} \frac{d_{\theta}\left(r, r_{p}\right)\left[1+d_{\theta}\left(l_{2 p}, l_{2 p+1}\right)\right]}{1+d_{\theta}\left(l_{2 p}, r\right)}+\lambda_{5} \frac{d_{\theta}\left(r, l_{2 p+1}\right)\left[1+d_{\theta}\left(l_{2 p}, r_{p}\right)\right]}{1+d_{\theta}\left(l_{2 p}, r\right)}
\end{gathered}
$$




$$
+\lambda_{6} \frac{d_{\theta}\left(l_{2 p}, r\right)\left[1+d_{\theta}\left(l_{2 p}, l_{2 p+1}\right)+d_{\theta}\left(r, l_{2 p+1}\right)\right]}{1+d_{\theta}\left(l_{2 p}, r\right)}+\mathbb{L} d_{\theta}\left(r, l_{2 p+1}\right) .
$$

We know that

, hence,

$$
d_{\theta}\left(r, r_{p}\right) \preceq \theta\left(r, r_{p}\right)\left[d_{\theta}\left(r, l_{2 p+1}\right)+d\left(l_{2 p+1}, r_{p}\right)\right]
$$

$$
\begin{aligned}
d_{\theta}\left(r, r_{p}\right) & \preceq \theta\left(r, r_{p}\right) d_{\theta}\left(l_{2 p+1}, r\right)+\theta\left(r, r_{p}\right) \lambda_{1} d_{\theta}\left(l_{2 p}, r\right)+\theta\left(r, r_{p}\right) \lambda_{2}\left[d_{\theta}\left(l_{2 p}, l_{2 p+1}\right)+d_{\theta}\left(r, r_{p}\right)\right] \\
& +\theta\left(r, r_{p}\right) \lambda_{3}\left[d_{\theta}\left(r, l_{2 p+1}\right)+d_{\theta}\left(l_{2 p}, r_{p}\right)\right]+\theta\left(r, r_{p}\right) \lambda_{4} \frac{d_{\theta}\left(r, r_{p}\right)\left[1+d_{\theta}\left(l_{2 p}, l_{2 p+1}\right)\right]}{1+d_{\theta}\left(l_{2 p}, r\right)} \\
& +\theta\left(r, r_{p}\right) \lambda_{5} \frac{d_{\theta}\left(r, l_{2 p+1}\right)\left[1+d_{\theta}\left(l_{2 p}, r_{p}\right)\right]}{1+d_{\theta}\left(l_{2 p}, r\right)}+\theta\left(r, r_{p}\right) \lambda_{6} \\
& \frac{d_{\theta}\left(l_{2 p}, r\right)\left[1+d_{\theta}\left(l_{2 p}, l_{2 p+1}\right)+\theta\left(r, r_{p}\right) d_{\theta}\left(r, l_{2 p+1}\right)\right]}{1+d_{\theta}\left(l_{2 p}, r\right)}+\theta\left(r, r_{p}\right) \mathbb{L} d_{\theta}\left(r, l_{2 p+1}\right) \\
\left|d_{\theta}\left(r, r_{p}\right)\right| & \leq\left|d_{\theta}\left(l_{2 p+1}, r\right)\right|+\lambda_{1}\left|d_{\theta}\left(l_{2 p}, r\right)\right|+\lambda_{2}\left[\left|d_{\theta}\left(l_{2 p}, l_{2 p+1}\right)\right|+\left|d_{\theta}\left(r, r_{p}\right)\right|\right]+\lambda_{3}\left[\left|d_{\theta}\left(r, l_{2 p+1}\right)\right|\right. \\
& \left.+\left|d_{\theta}\left(l_{2 p}, r_{p}\right)\right|\right]+\lambda_{4}\left|\frac{d_{\theta}\left(r, r_{p}\right)\left[1+\mid d_{\theta}\left(l_{2 p}, l_{2 p+1}\right)\right]}{1+d_{\theta}\left(l_{2 p}, r\right)}\right|+\lambda_{5}\left|\frac{d_{\theta}\left(r, l_{2 p+1}\right)\left[1+d_{\theta}\left(l_{2 p}, r_{p}\right)\right]}{1+d_{\theta}\left(l_{2 p}, r\right)}\right| \\
& +\lambda_{6}\left|\frac{d_{\theta}\left(l_{2 p}, r\right)\left[1+d_{\theta}\left(l_{2 p}, l_{2 p+1}\right)+d_{\theta}\left(r, l_{2 p+1}\right)\right]}{1+d_{\theta}\left(l_{2 p}, r\right)}\right|+\mathbb{L}\left|d_{\theta}\left(r, l_{2 p+1}\right)\right| .
\end{aligned}
$$

By letting $p \rightarrow \infty$ in the above expression, we get

$$
\left|d_{\theta}\left(r, r_{p}\right)\right| \rightarrow 0 \text { as } p \rightarrow \infty \text {. }
$$

By Lemma (2.4), $r_{p} \rightarrow r$ as $p \rightarrow \infty$; also since $\mathbb{T} r$ is closed, thus $r \in \mathbb{T} r$. Similarly, we have $r \in \mathbb{S} r$. Hence, $\mathrm{S}$ and $\mathbb{T}$ have a common fixed point.

In what follows, we deduce some consequences of Theorem 2.1. For $\lambda_{6}=0$, we have

Corollary 2.1. Let $\left(\mathbb{G}, d_{\theta}\right)$ be a complete complex valued extended b-metric space, $\theta: \mathbb{G} \times \mathbb{G} \rightarrow[1, \infty)$ and $\mathbb{S}, \mathbb{T}: \mathbb{G} \times \mathbb{G} \rightarrow$ $C B(G)$ be a pair of multi-valued mappings with g.l.b property such that

$$
\begin{aligned}
& \lambda_{1} d_{\theta}(l, m)+\lambda_{2}\left[d_{\theta}(l, \mathbb{S} l)+d_{\theta}(m, \mathbb{T} m)\right]+\lambda_{3}\left[d_{\theta}(m, \mathbb{S} l)+d_{\theta}(l, \mathbb{T} m)\right] \\
& +\lambda_{4} \frac{d_{\theta}(m, \mathbb{T} m)\left[1+d_{\theta}(l, S l)\right]}{1+d_{\theta}(l, m)}+\lambda_{5} \frac{d_{\theta}(m, \mathbf{S} l)\left[1+d_{\theta}(l, \mathbb{T} m)\right]}{1+d_{\theta}(l, m)}+\mathbb{L} d_{\theta}(m, \mathbb{T} l) \in s(\mathbb{S} l, \mathbb{T} m),
\end{aligned}
$$

for all $l, m \in \mathbb{G}$ and $\lambda_{1}, \lambda_{2}, \lambda_{3}, \lambda_{4}, \lambda_{5}, \mathbb{L}$ are nonnegative real numbers with $\lambda_{1}+2 \lambda_{2}+2 \lambda_{3} \theta\left(l_{0}, l_{2}\right)+\lambda_{4}<1, k\left(1-\lambda_{2}-\right.$ $\left.\lambda_{3} \theta\left(l_{0}, l_{2}\right)-\lambda_{4}\right)=\left(\lambda_{1}+\lambda_{2}+\lambda_{3} \theta\left(l_{0}, l_{2}\right)\right)$. Then $\mathbf{S}$ and $\mathbb{T}$ have a common fixed point.

For $\lambda_{i}=0$, where $i=5,6$, we have the next corollary.

Corollary 2.2. Let $\left(\mathbb{G}, d_{\theta}\right)$ be a complete complex valued extended b-metric space, $\theta: \mathbb{G} \times \mathbb{G} \rightarrow[1, \infty)$ and $S, \mathbb{T}: \mathbb{G} \times \mathbb{G} \rightarrow$ $C B(G)$ be a pair of multi-valued mappings with g.l.b property such that

$$
\begin{aligned}
& \lambda_{1} d_{\theta}(l, m)+\lambda_{2}\left[d_{\theta}(l, \mathbf{S} l)+d_{\theta}(m, \mathbb{T} m)\right]+\lambda_{3}\left[d_{\theta}(m, \mathbb{S} l)+d_{\theta}(l, \mathbb{T} m)\right] \\
& +\lambda_{4} \frac{d_{\theta}(m, \mathbb{T} m)\left[1+d_{\theta}(l, \mathbb{S} l)\right]}{1+d_{\theta}(l, m)}+\mathbb{L} d_{\theta}(m, \mathbb{T} l) \in s(\mathbb{S} l, \mathbb{T} m),
\end{aligned}
$$

for all $l, m \in \mathbb{G}$ and $\lambda_{1}, \lambda_{2}, \lambda_{3}, \lambda_{4}, \mathbb{L}$ are nonnegative real numbers with $\lambda_{1}+2 \lambda_{2}+2 \lambda_{3} \theta\left(l_{0}, l_{2}\right)+\lambda_{4}<1, k\left(1-\lambda_{2}-\right.$ $\left.\lambda_{3} \theta\left(l_{o}, l_{2}\right)-\lambda_{4}\right)=\left(\lambda_{1}+\lambda_{2}+\lambda_{3} \theta\left(l_{0}, l_{2}\right)\right)$. Then $\mathbf{S}$ and $\mathbb{T}$ have a common fixed point.

For $\lambda_{i}=0$, where $i=4,5,6$, we have the following result. follow: 
Corollary 2.3. Let $\left(\mathbb{G}, d_{\theta}\right)$ be a complete complex valued extended b-metric space, $\theta: \mathbb{G} \times \mathbb{G} \rightarrow[1, \infty)$ and $S, \mathbb{T}: \mathbb{G} \times \mathbb{G} \rightarrow$ $C B(\mathbb{G})$ be a pair of multi-valued mappings with g.l.b property such that

$$
\lambda_{1} d_{\theta}(l, m)+\lambda_{2}\left[d_{\theta}(l, \mathbb{S} l)+d_{\theta}(m, \mathbb{T} m)\right]+\lambda_{3}\left[d_{\theta}(m, \mathbb{S} l)+d_{\theta}(l, \mathbb{T} m)\right]+\mathbb{L} d_{\theta}(m, \mathbb{T} l) \in s(\mathbb{S} l, \mathbb{T} m),
$$

for all $l, m \in \mathbb{G}$ and $\lambda_{1}, \lambda_{2}, \lambda_{3}, \mathbb{L}$ are nonnegative real numbers with $\lambda_{1}+2 \lambda_{2}+2 \lambda_{3} \theta\left(l_{o}, l_{2}\right)<1$. $k\left(1-\lambda_{2}-\lambda_{3} \theta\left(l_{0}, l_{2}\right)=\right.$ $\left(\lambda_{1}+\lambda_{2}+\lambda_{3} \theta\left(l_{0}, l_{2}\right)\right)$. Then $\mathbb{S}$ and $\mathbb{T}$ have a common fixed point.

For $\mathrm{S}=\mathbb{T}$, we have the following corollary.

Corollary 2.4. Let $\left(\mathbb{G}, d_{\theta}\right)$ be a complete complex valued extended b-metric space, $\theta: \mathbb{G} \times \mathbb{G} \rightarrow[1, \infty)$ and $\mathbb{T}: \mathbb{G} \rightarrow C B(\mathbb{G})$ be a multi-valued mapping with g.l.b property such that

$$
\begin{aligned}
& \lambda_{1} d_{\theta}(l, m)+\lambda_{2}\left[d_{\theta}(l, \mathbb{T} l)+d_{\theta}(m, \mathbb{T} m)\right]+\lambda_{3}\left[d_{\theta}(m, \mathbb{T} l)+d_{\theta}(l, \mathbb{T} m)\right] \\
& +\lambda_{4} \frac{d_{\theta}(m, \mathbb{T} m)\left[1+d_{\theta}(l, \mathbb{T} l)\right]}{1+d_{\theta}(l, m)}+\lambda_{5} \frac{d_{\theta}(m, \mathbb{T} l)\left[1+d_{\theta}(l, \mathbb{T} m)\right]}{1+d_{\theta}(l, m)} \\
& +\lambda_{6} \frac{d_{\theta}(l, m)\left[1+d_{\theta}(l, \mathbb{T} l)+d_{\theta}(m, \mathbb{T} l)\right]}{1+d_{\theta}(l, m)}+\mathbb{L} d_{\theta}(m, \mathbb{T} l) \in s(\mathbb{T} l, \mathbb{T} m)
\end{aligned}
$$

for all $l, m \in \mathbb{G}$ and $\lambda_{1}, \lambda_{2}, \lambda_{3}, \lambda_{4}, \lambda_{5}, \lambda_{6}, \mathbb{L}$ are nonnegative real numbers with $\lambda_{1}+2 \lambda_{2}$

$+2 \lambda_{3} \theta(l, m)+\lambda_{4}+\lambda_{5}+\lambda_{6}<1, k\left(1-\lambda_{2}-\lambda_{3} \theta(l, m)-\lambda_{4}\right)=\left(\lambda_{1}+\lambda_{2}+\lambda_{3} \theta(l, m)+\lambda_{6}\right)$ where $k \in[0, \infty)$ be such that for each $l_{0} \in \mathbb{G} \lim _{p, q \rightarrow \infty} \theta\left(l_{p}, l_{q}\right)<\frac{1}{k}$. Then $\mathbb{T}$ has a fixed point.

Remark 2.1. From Corollary 4.6, we can deduce several corollaries by putting $\lambda_{6}=0, \lambda_{6}, \lambda_{5}=0$ and $\lambda_{6}, \lambda_{5}, \lambda_{4}=0$.

Now, we provide an example to validate the hypotheses of Theorem 2.1 as follows.

Example 2.1. Let $\mathbb{G}=[0, \infty)$. Define $\theta: \mathbb{G} \times \mathbb{G} \longrightarrow[1, \infty)$ by

$$
\theta(l, m)=\frac{5+l+m}{1+l+m}, \text { for all } l, m \in \mathbb{G},
$$

and $d_{\theta}: \mathbb{G} \times \mathbb{G} \longrightarrow \mathbb{C}$ by

$$
d_{\theta}(l, m)=|l-m|^{2}+i|l-m|^{2}, \text { for all } l, m \in \mathbb{G} .
$$

Then $\left(\mathbb{G}, d_{\theta}\right)$ is a complete complex-valued extended b-metric space. Consider the mappings $\mathbb{S}, \mathbb{T}: \mathbb{G} \longrightarrow C B(\mathbb{G})$, defined by

$$
\begin{aligned}
& \mathbb{S} l= \begin{cases}{\left[0, \frac{l}{7}\right],} & \text { if } l \in[0,1] \\
{[3 l, 5 l],} & \text { otherwise. }\end{cases} \\
& \mathbb{T} l= \begin{cases}{\left[0, \frac{l}{14}\right],} & \text { if } l \in[0,1] \\
{[5 l, 9 l],} & \text { otherwise. }\end{cases}
\end{aligned}
$$

If $l=m=0$, the contractive condition of Theorem 2.1 holds trivially. Assume without loss of generality that both $l$ and $m$ are nonzero with $l<m$. Then

$$
\begin{gathered}
d_{\theta}(l, \mathrm{~S} l)=\left|l-\frac{l}{7}\right|^{2}+i\left|l-\frac{l}{7}\right|^{2}, d_{\theta}(m, \mathbb{T} m)=\left|m-\frac{m}{14}\right|^{2}+i\left|m-\frac{m}{14}\right|^{2}, \\
d_{\theta}(m, \mathrm{~S} l)=\left|m-\frac{l}{7}\right|^{2}+i\left|m-\frac{l}{7}\right|^{2}, d_{\theta}(l, \mathbb{T} m)=\left|l-\frac{m}{14}\right|^{2}+i\left|l-\frac{m}{14}\right|^{2}, \\
s(\mathrm{~S} l, \mathbb{T} m)=s\left(\left|\frac{l}{7}-\frac{m}{14}\right|^{2}+i\left|\frac{l}{7}-\frac{m}{14}\right|^{2}\right) .
\end{gathered}
$$

By taking $\lambda_{2}=\lambda_{3}=\lambda_{4}=\lambda_{5}=\lambda_{6}=0$ and $\lambda_{1}=\frac{1}{2}$, it can be verified directly that all the conditions of Theorem 2.1 are satisfied. In this case, 0 is a common fixed point of $\mathrm{S}$ and $\mathbb{T}$. 


\section{Banach Type Contractive Mapping}

Theorem 3.1. Let $\left(\mathbb{G}, d_{\theta}\right)$ be a complete $C . V$ extended b-metric space, $\theta: \mathbb{G} \times \mathbb{G} \rightarrow[1, \infty)$ be a function and $\mathbb{S}, \mathbb{T}: \mathbb{G} \rightarrow$ $C B(G)$ be a pair multi-valued mappings satisfying the g.l.b property such that

$$
\lambda_{1} d_{\theta}(l, m)+\frac{\lambda_{2} d_{\theta}(l, \mathbf{S} l) d_{\theta}(m, \mathbb{T} m)+\lambda_{3} d_{\theta}(m, \mathbf{S} l) d_{\theta}(l, \mathbb{T} m)}{1+d_{\theta}(l, m)} \in s(\mathbb{S} l, \mathbb{T} m),
$$

for all $l, m \in \mathbb{G}$ and $\lambda_{1}, \lambda_{2}, \lambda_{3}$ are non negative real numbers with $\lambda_{1}+\lambda_{2}+\lambda_{3}<1$, and assumed that $k\left(1-\lambda_{2}\right)=\lambda_{1}$ where $k \in[0,1)$ for each $l_{o} \in \mathbb{G}, \lim _{p, q \rightarrow \infty} \theta\left(l_{p}, m_{q}\right) k<1$. Then $\mathbf{S}$ and $\mathbb{T}$ have a common fixed point.

Proof. Let $l_{o} \in \mathrm{G}$, then $T l_{o}$ is not empty; so we can take $l_{1} \in T l_{0}$. Thus, from (3.1),

$$
\lambda_{1} d_{\theta}\left(l_{o}, l_{1}\right)+\frac{\lambda_{2} d_{\theta}\left(l_{o}, S l_{o}\right) d_{\theta}\left(l_{1}, \mathbb{T} l_{1}\right)+\lambda_{3} d_{\theta}\left(l_{1}, S l_{o}\right) d_{\theta}\left(l_{o}, \mathbb{T} l_{1}\right)}{1+d_{\theta}\left(l_{0}, l_{1}\right)} \in s\left(\mathbb{S} l_{o}, \mathbb{T} l_{1}\right) .
$$

This implies that

$$
\begin{gathered}
\lambda_{1} d_{\theta}\left(l_{o}, l_{1}\right)+\frac{\lambda_{2} d_{\theta}\left(l_{o}, S l_{o}\right) d_{\theta}\left(l_{1}, \mathbb{T} l_{1}\right)+\lambda_{3} d_{\theta}\left(l_{1}, S l_{o}\right) d_{\theta}\left(l_{o}, \mathbb{T} l_{1}\right)}{1+d_{\theta}\left(l_{0}, l_{1}\right)} \in \bigcap_{a^{\prime} \in S l_{o}} s\left(a^{\prime}, \mathbb{T} l_{1}\right), \\
\lambda_{1} d_{\theta}\left(l_{o}, l_{1}\right)+\frac{\lambda_{2} d_{\theta}\left(l_{o}, S l_{o}\right) d_{\theta}\left(l_{1}, \mathbb{T} l_{1}\right)+\lambda_{3} d_{\theta}\left(l_{1}, S l_{o}\right) d_{\theta}\left(l_{o}, \mathbb{T} l_{1}\right)}{1+d_{\theta}\left(l_{0}, l_{1}\right)} \in s\left(a^{\prime}, \mathbb{T} l_{1}\right), \quad \forall a^{\prime} \in \mathbb{S} l_{o} .
\end{gathered}
$$

Since $l_{1} \in \mathbb{S} l_{0}$, we have

$$
\begin{gathered}
\lambda_{1} d_{\theta}\left(l_{o}, l_{1}\right)+\frac{\lambda_{2} d_{\theta}\left(l_{o}, S l_{o}\right) d_{\theta}\left(l_{1}, \mathbb{T} l_{1}\right)+\lambda_{3} d_{\theta}\left(l_{1}, S l_{o}\right) d_{\theta}\left(l_{o}, \mathbb{T} l_{1}\right)}{1+d_{\theta}\left(l_{0}, l_{1}\right)} \in s\left(l_{1}, \mathbb{T} l_{1}\right) \\
\lambda_{1} d_{\theta}\left(l_{o}, l_{1}\right)+\frac{\lambda_{2} d_{\theta}\left(l_{o}, S l_{o}\right) d_{\theta}\left(l_{1}, \mathbb{T} l_{1}\right)+\lambda_{3} d_{\theta}\left(l_{1}, S l_{o}\right) d_{\theta}\left(l_{o}, \mathbb{T} l_{1}\right)}{1+d_{\theta}\left(l_{0}, l_{1}\right)} \in \bigcup_{b^{\prime} \in \mathbb{T} l_{1}} s\left(d_{\theta}\left(l_{1}, b^{\prime}\right)\right) .
\end{gathered}
$$

Therefore, there exist $l_{2} \in \mathbb{T} l_{2}$ such that

$$
\lambda_{1} d_{\theta}\left(l_{o}, l_{1}\right)+\frac{\lambda_{2} d_{\theta}\left(l_{o}, S l_{o}\right) d_{\theta}\left(l_{1}, \mathbb{T} l_{1}\right)+\lambda_{3} d_{\theta}\left(l_{1}, S l_{o}\right) d_{\theta}\left(l_{o}, \mathbb{T} l_{1}\right)}{1+d_{\theta}\left(l_{0}, l_{1}\right)} \in s\left(d_{\theta}\left(l_{1}, l_{2}\right)\right) .
$$

By definition and the g.l.b property of $\mathrm{S}$ and $\mathbb{T}$, we get

$$
\begin{gathered}
d_{\theta}\left(l_{1}, l_{2}\right) \preceq \lambda_{1} d_{\theta}\left(l_{o}, l_{1}\right)+\frac{\lambda_{2} d_{\theta}\left(l_{o}, S l_{o}\right) d_{\theta}\left(l_{1}, \mathbb{T} l_{1}\right)+\lambda_{3} d_{\theta}\left(l_{1}, S l_{o}\right) d_{\theta}\left(l_{o}, \mathbb{T} l_{1}\right)}{1+d_{\theta}\left(l_{0}, l_{1}\right)}, \\
d_{\theta}\left(l_{1}, l_{2}\right) \preceq \lambda_{1} d_{\theta}\left(l_{o}, l_{1}\right)+\frac{\lambda_{2} d_{\theta}\left(l_{o}, l_{1}\right) d_{\theta}\left(l_{1}, l_{2}\right)+\lambda_{3} d_{\theta}\left(l_{1}, l_{1}\right) d_{\theta}\left(l_{o}, l_{2}\right)}{1+d_{\theta}\left(l_{0}, l_{1}\right)} .
\end{gathered}
$$

This implies

$$
\begin{aligned}
\left|d_{\theta}\left(l_{1}, l_{2}\right)\right| & \leq \lambda_{1}\left|d_{\theta}\left(l_{o}, l_{1}\right)\right|+\frac{\lambda_{2}\left|d_{\theta}\left(l_{0}, l_{1}\right)\right|\left|d_{\theta}\left(l_{1}, l_{2}\right)\right|}{1+\left|d_{\theta}\left(l_{0}, l_{1}\right)\right|} \\
& =\lambda_{1}\left|d_{\theta}\left(l_{o}, l_{1}\right)\right|+\lambda_{2}\left|d_{\theta}\left(l_{1}, l_{2}\right)\right| \frac{\left|d_{\theta}\left(l_{0}, l_{1}\right)\right|}{\left|1+d_{\theta}\left(l_{0}, l_{1}\right)\right|}
\end{aligned}
$$

Or

$$
\begin{aligned}
\left|d_{\theta}\left(l_{1}, l_{2}\right)\right| & \leq \lambda_{1}\left|d_{\theta}\left(l_{0}, l_{1}\right)\right|+\lambda_{2}\left|d_{\theta}\left(l_{1}, l_{2}\right)\right| \\
\left(1-\lambda_{2}\right)\left|d_{\theta}\left(l_{1}, l_{2}\right)\right| & \leq \lambda_{1}\left|d_{\theta}\left(l_{0}, l_{1}\right)\right| \\
\left|d_{\theta}\left(l_{1}, l_{2}\right)\right| & \leq \frac{\lambda_{1}}{\left(1-\lambda_{2}\right)}\left|d_{\theta}\left(l_{o}, l_{1}\right)\right| .
\end{aligned}
$$


Inductively, we develop a sequence $\left\{l_{p}\right\}$ in $\mathbb{G}$ such that

$$
\begin{aligned}
\left|d_{\theta}\left(l_{1}, l_{2}\right)\right| & \leq k\left|d_{\theta}\left(l_{0}, l_{1}\right)\right| . \\
\left|d_{\theta}\left(l_{2}, l_{3}\right)\right| & \leq k^{2}\left|d_{\theta}\left(l_{0}, l_{1}\right)\right| . \\
\cdot & \cdot \\
\cdot & \\
\left|d_{\theta}\left(l_{p}, l_{p+1}\right)\right| & \leq k^{p}\left|d_{\theta}\left(l_{o}, l_{1}\right)\right| .
\end{aligned}
$$

Now, by triangular inequality, for $q>p$, we have

$$
\begin{aligned}
d_{\theta}\left(l_{p}, l_{q}\right) & \preceq \theta\left(l_{p}, l_{q}\right) k^{p} d_{\theta}\left(l_{0}, l_{1}\right)+\theta\left(l_{p}, l_{q}\right) \theta\left(l_{p+1}, l_{q}\right) k^{p+1} d_{\theta}\left(l_{o}, l_{1}\right)+\cdots \\
& +\theta\left(l_{p}, l_{q}\right) \theta\left(l_{p+1}, l_{q}\right) \cdots \theta\left(l_{q-2}, l_{q}\right) \theta\left(l_{q-1}, l_{q}\right) k^{q-1} d_{\theta}\left(l_{o}, l_{1}\right) \\
d_{\theta}\left(l_{p}, l_{q}\right) & \preceq d_{\theta}\left(l_{0}, l_{1}\right)\left[\theta\left(l_{p}, l_{q}\right) k^{p}+\theta\left(l_{p}, l_{q}\right) \theta\left(l_{p+1}, l_{q}\right) k^{p+1}+\cdots\right. \\
& \left.+\theta\left(l_{p}, l_{q}\right) \theta\left(l_{p+1}, l_{q}\right) \ldots \theta\left(l_{q-2}, l_{q}\right) \theta\left(l_{q-1}, l_{p}\right) k^{q-1}\right] .
\end{aligned}
$$

Since $\lim _{p, q \rightarrow \infty} \theta\left(l_{p}, l_{q}\right) k<1$, so the series $\sum_{p=1}^{\infty} k^{p} \prod_{i=1}^{p} \theta\left(l_{i}, l_{q}\right)$ converges by ratio test for each $q \in \mathbb{N}$. Let

$$
S=\sum_{p=1}^{\infty} k^{p} \prod_{i=1}^{p} \theta\left(l_{i}, l_{q}\right), S_{p}=\sum_{j=1}^{p} k^{j} \prod_{i=1}^{p} \theta\left(l_{i}, l_{q}\right)
$$

Thus, for $q>p$, the above can be written as

$$
d_{\theta}\left(l_{p}, l_{q}\right) \preceq d_{\theta}\left(l_{o}, l_{1}\right)\left[S_{q-1}-S_{P}\right]
$$

In other words,

$$
\left|d_{\theta}\left(l_{p}, l_{q}\right)\right| \leq\left|d_{\theta}\left(l_{o}, l_{1}\right)\right|\left[S_{q-1}-S_{p}\right]
$$

Now, by taking $p \rightarrow \infty$, we get

$$
\left|d_{\theta}\left(l_{p}, l_{q}\right)\right| \rightarrow 0
$$

By lemma (2.4), we conclude that $\left\{l_{p}\right\}$ is a Cauchy sequence. Since $G$ is complete, then, there exists an element $r$ such that $l_{p} \rightarrow r \in \mathbb{G}$ as $p \rightarrow \infty$.

Now, to show $r \in \mathbb{S} r$ and $r \in \mathbb{T} r$. For this, we have from (5.1)

$$
\begin{gathered}
\lambda_{1} d_{\theta}\left(l_{2 p}, r\right)+\frac{\lambda_{2} d_{\theta}\left(l_{2 p}, \mathbb{S} l_{2 p}\right) d_{\theta}(r, \mathbb{T} r)+\lambda_{3} d_{\theta}\left(r, S l_{2 p}\right) d_{\theta}\left(l_{2 p}, \mathbb{T} r\right)}{1+d_{\theta}\left(l_{2 p}, r\right)} \in s\left(\mathbb{S} l_{2 p}, \mathbb{T} r\right), \\
\lambda_{1} d_{\theta}\left(l_{2 p}, r\right)+\frac{\lambda_{2} d_{\theta}\left(l_{2 p}, S l_{2 p}\right) d_{\theta}(r, \mathbb{T} r)+\lambda_{3} d_{\theta}\left(r, S l_{2 p}\right) d_{\theta}\left(l_{2 p}, \mathbb{T} r\right)}{1+d_{\theta}\left(l_{2 p}, r\right)} \in \bigcap_{a^{\prime} \in S l_{2 p}} s\left(a^{\prime}, \mathbb{T} r\right) .
\end{gathered}
$$

Since $l_{2 p+1} \in S l_{2 p}$, we have

$$
\begin{gathered}
\lambda_{1} d_{\theta}\left(l_{2 p}, r\right)+\frac{\lambda_{2} d_{\theta}\left(l_{2 p}, \mathbb{S} l_{2 p}\right) d_{\theta}(r, \mathbb{T} r)+\lambda_{3} d_{\theta}\left(r, S l_{2 p}\right) d_{\theta}\left(l_{2 p}, \mathbb{T} r\right)}{1+d_{\theta}\left(l_{2 p}, r\right)} \in s\left(l_{2 p+1}, \mathbb{T} r\right), \\
\lambda_{1} d_{\theta}\left(l_{2 p}, r\right)+\frac{\lambda_{2} d_{\theta}\left(l_{2 p}, \mathbb{S} l_{2 p}\right) d_{\theta}(r, \mathbb{T} r)+\lambda_{3} d_{\theta}\left(r, \mathbb{S} l_{2 p}\right) d_{\theta}\left(l_{2 p}, \mathbb{T} r\right)}{1+d_{\theta}\left(l_{2 p}, r\right)} \in \bigcup_{b^{\prime} \in \mathbb{T} r} s\left(d_{\theta}\left(l_{2 p+1}, b^{\prime}\right)\right) .
\end{gathered}
$$

This implies that there exists $r_{p} \in \mathbb{T} r$ such that

$$
\lambda_{1} d_{\theta}\left(l_{2 p}, r\right)+\frac{\lambda_{2} d_{\theta}\left(l_{2 p}, S l_{2 p}\right) d_{\theta}(r, \mathbb{T} r)+\lambda_{3} d_{\theta}\left(r, S l_{2 p}\right) d_{\theta}\left(l_{2 p}, \mathbb{T} r\right)}{1+d_{\theta}\left(l_{2 p}, r\right)} \in s\left(d_{\theta}\left(l_{2 p+1}, r_{p}\right)\right) .
$$


That is,

$$
d_{\theta}\left(l_{2 p+1}, r_{p}\right) \preceq \lambda_{1} d_{\theta}\left(l_{2 p}, r\right)+\frac{\lambda_{2} d_{\theta}\left(l_{2 p}, l_{2 p+1}\right) d_{\theta}\left(r, r_{p}\right)+\lambda_{3} d_{\theta}\left(r, S l_{2 p}\right) d_{\theta}\left(l_{2 p}, \mathbb{T} r\right)}{1+d_{\theta}\left(l_{2 p}, r\right)}
$$

Now,

$$
\begin{gathered}
d_{\theta}\left(r, r_{p}\right) \preceq \theta\left(r, r_{p}\right)\left[d_{\theta}\left(r, l_{2 p+1}\right)+d_{\theta}\left(l_{2 p+1}, r_{p}\right)\right] \\
d_{\theta}\left(r, r_{p}\right) \preceq \theta\left(r, r_{p}\right) d_{\theta}\left(r, l_{2 p+1}\right)+\theta\left(r, r_{p}\right) \lambda_{1} d_{\theta}\left(l_{2 p}, r\right) \\
+\frac{\theta\left(r, r_{p}\right) \lambda_{2} d_{\theta}\left(l_{2 p}, l_{2 p+1}\right) d_{\theta}\left(r, r_{p}\right)+\theta\left(r, r_{p}\right) \lambda_{3} d_{\theta}\left(r, l_{2 p+1}\right) d_{\theta}\left(l_{2 p}, r_{p}\right)}{1+d_{\theta}\left(l_{2 p}, r\right)} .
\end{gathered}
$$

It follows that

$$
\begin{aligned}
\left|d_{\theta}\left(r, r_{p}\right)\right| & \leq \theta\left(r, r_{p}\right)\left|d_{\theta}\left(r, l_{2 p+1}\right)\right|+\theta\left(r, r_{p}\right) \lambda_{1}\left|d_{\theta}\left(l_{2 p}, r\right)\right| \\
& +\frac{\theta\left(r, r_{p}\right) \lambda_{2}\left|d_{\theta}\left(l_{2 p}, l_{2 p+1}\right)\right|\left|d_{\theta}\left(r, r_{p}\right)\right|+\theta\left(r, r_{p}\right) \lambda_{3}\left|d_{\theta}\left(r, l_{2 p+1}\right)\right|\left|d_{\theta}\left(l_{2 p}, r_{p}\right)\right|}{1+\left|d_{\theta}\left(l_{2 p}, r\right)\right|} .
\end{aligned}
$$

Letting $p \rightarrow \infty$, we get $\left|d_{\theta}\left(r, r_{p}\right)\right| \rightarrow 0$.

By using Lemma 2.4, we have $r_{p} \rightarrow r$. Since $\mathbb{T} r$ is closed, so $r \in \mathbb{T} r$. On similar steps, we can prove that $r \in \mathrm{S} r$. Thus, $\mathbb{T}$ and $\mathrm{S}$ have a common fixed point.

By putting $\lambda_{3}=0$ in Theorem 3.1, we have the next result.

Corollary 3.1. Let $\left(\mathbb{G}, d_{\theta}\right)$ ba a complete $C$. V extended b-metric $\theta: \mathbb{G} \times \mathbb{G} \rightarrow[1, \infty)$ be a function, and $\mathbb{S}, \mathbb{T}: \mathbb{G} \rightarrow C B(\mathbb{G})$ be a pair of multi-valued mappings satisfying the g.l.b property such that

$$
\lambda_{1} d_{\theta}(l, m)+\frac{\lambda_{2} d_{\theta}(l, \mathbb{S} l) d_{\theta}(m, \mathbb{T} m)}{1+d_{\theta}(l, m)} \in s(\mathbf{S} l, \mathbb{T} m),
$$

for all $l, m \in \mathbb{G}$ and $\lambda_{1}, \lambda_{2}$ are non negative reals with $\lambda_{1}+\lambda_{2}<1$ and assumed $k\left(1-\lambda_{2}\right)=\lambda_{1}$ where $k \in[0,1)$ such that for each $l_{o} \in \mathbb{G}, \lim _{p, q \rightarrow \infty} \theta\left(l_{p}, l_{q}\right) k<1$. Then, $\mathrm{S}$ and $\mathbb{T}$ have a common fixed point.

By putting $\mathrm{S}=\mathbb{T}$ in Theorem 3.1, we have the following. corollary.

Corollary 3.2. Let $\left(\mathbb{G}, d_{\theta}\right)$ be a complete C.V extended b-metric space, $\theta: \mathbb{G} \times \mathbb{G} \rightarrow[1, \infty)$ be a function, and $\mathbb{T}: \mathbb{G} \rightarrow$ $C B(\mathbb{G})$ be a multi-valued mapping satisfying the g.l.b property such that

$$
\lambda_{1} d_{\theta}(l, m)+\frac{\lambda_{2} d_{\theta}(l, \mathbb{T} l) d_{\theta}(m, \mathbb{T} m)+\lambda_{3} d_{\theta}(m, \mathbb{T} l) d_{\theta}(l, \mathbb{T} m)}{1+d_{\theta}(l, m)} \in s(\mathbb{T} l, \mathbb{T} m)
$$

for all $l, m \in \mathbb{G}$ and $\lambda_{1}, \lambda_{2}, \lambda_{3}$ are non negative reals with $\lambda_{1}+\lambda_{2}+\lambda_{3}<1$ and assumed that $k\left(1-\lambda_{2}\right)=\lambda_{1}$ where $k \in[0,1)$ for each, $l_{o} \in \mathbb{G} \lim _{p, q \rightarrow \infty} \theta\left(l_{p}, m_{q}\right) k<1$. Then $\mathbb{T}$ has a fixed point.

\section{Kannan Type Contractive Mapping}

Theorem 4.1. Let $\left(\mathbb{G}, d_{\theta}\right)$ be a complete C.V extended b-metric space and $\theta: \mathbb{G} \times \mathbb{G} \rightarrow[1, \infty)$ be a function. Let $\mathrm{S}, \mathbb{T}: \mathbb{G} \rightarrow C B(\mathbb{G})$ be a pair of multi-valued mappings satisfying the g.l.b property such that

$$
\lambda_{1} d_{\theta}(l, \mathbf{S} l)+\lambda_{2} d_{\theta}(m, \mathbb{T} m)+\frac{\lambda_{3} d_{\theta}(l, \mathbb{S} l) d_{\theta}(m, \mathbb{T} m)}{1+d_{\theta}(l, m)} \in s(\mathbf{S} l, \mathbb{T} m),
$$

for all $l, m \in \mathbb{G}$ and $\lambda_{1}, \lambda_{2}, \lambda_{3}$ are non negative reals with $\lambda_{1}+\lambda_{2}+\lambda_{3}<1$ and assumed $k\left(1-\lambda_{2}-\lambda_{3}\right)=\lambda_{1}$, where $k \in[0,1)$ is such that for each $l_{o} \in \mathbb{G}, \lim _{p, q \rightarrow \infty} \theta\left(l_{p}, l_{q}\right) k<1$. Then $\mathbb{S}$ and $\mathbb{T}$ have a common fixed point. 
Proof. Let $l_{o} \in \mathbb{G}$, then $T l_{o}$ is non-empty; so we can take $l_{1} \in T l_{o}$. Thus from (4.1),

$$
\begin{aligned}
& \lambda_{1} d_{\theta}\left(l_{0}, S l_{o}\right)+\lambda_{2} d_{\theta}\left(l_{1}, \mathbb{T} l_{1}\right)+\frac{\lambda_{3} d_{\theta}\left(l_{o}, S l_{o}\right) d_{\theta}\left(l_{1}, \mathbb{T} l_{1}\right)}{1+d_{\theta}\left(l_{0}, l_{1}\right)} \in s\left(\mathbb{S} l_{o}, \mathbb{T} l_{1}\right), \\
& \lambda_{1} d_{\theta}\left(l_{o}, S l_{o}\right)+\lambda_{2} d_{\theta}\left(l_{1}, \mathbb{T} l_{1}\right)+\frac{\lambda_{3} d_{\theta}\left(l_{0}, S l_{o}\right) d_{\theta}\left(l_{1}, \mathbb{T} l_{1}\right)}{1+d_{\theta}\left(l_{o}, l_{1}\right)} \in \bigcap_{a^{\prime} \in \mathbb{S} l_{o}} s\left(a^{\prime}, \mathbb{T} l_{1}\right), \\
& \lambda_{1} d_{\theta}\left(l_{o}, S l_{o}\right)+\lambda_{2} d_{\theta}\left(l_{1}, \mathbb{T} l_{1}\right)+\frac{\lambda_{3} d_{\theta}\left(l_{o}, \mathbb{S} l_{o}\right) d_{\theta}\left(l_{1}, \mathbb{T} l_{1}\right)}{1+d_{\theta}\left(l_{o}, l_{1}\right)} \in s\left(a^{\prime}, \mathbb{T} l_{1}\right), \quad \forall a^{\prime} \in \mathbb{S} l_{o} .
\end{aligned}
$$

Since $l_{1} \in S l_{0}$, thus

$$
\begin{gathered}
\lambda_{1} d_{\theta}\left(l_{o}, S l_{o}\right)+\lambda_{2} d_{\theta}\left(l_{1}, \mathbb{T} l_{1}\right)+\frac{\lambda_{3} d_{\theta}\left(l_{o}, S l_{o}\right) d_{\theta}\left(l_{1}, \mathbb{T} l_{1}\right)}{1+d_{\theta}\left(l_{o}, l_{1}\right)} \in s\left(l_{1}, \mathbb{T} l_{1}\right), \\
\lambda_{1} d_{\theta}\left(l_{o}, S l_{o}\right)+\lambda_{2} d_{\theta}\left(l_{1}, \mathbb{T} l_{1}\right)+\frac{\lambda_{3} d_{\theta}\left(l_{o}, S l_{o}\right) d_{\theta}\left(l_{1}, \mathbb{T} l_{1}\right)}{1+d_{\theta}\left(l_{o}, l_{1}\right)} \in \bigcup_{b^{\prime} \in \mathbb{T} l_{1}} s\left(d_{\theta}\left(l_{1}, b^{\prime}\right)\right) .
\end{gathered}
$$

Therefore, there exist $l_{2} \in \mathbb{T} l_{1}$ such that

$$
\lambda_{1} d_{\theta}\left(l_{o}, S l_{o}\right)+\lambda_{2} d_{\theta}\left(l_{1}, \mathbb{T} l_{1}\right)+\frac{\lambda_{3} d_{\theta}\left(l_{o}, S l_{o}\right) d_{\theta}\left(l_{1}, \mathbb{T} l_{1}\right)}{1+d_{\theta}\left(l_{o}, l_{1}\right)} \in s\left(d_{\theta}\left(l_{1}, l_{2}\right)\right) .
$$

By definition, we get

$$
d_{\theta}\left(l_{1}, l_{2}\right) \preceq \lambda_{1} d_{\theta}\left(l_{o}, \mathbb{S} l_{o}\right)+\lambda_{2} d_{\theta}\left(l_{1}, \mathbb{T} l_{1}\right)+\frac{\lambda_{3} d_{\theta}\left(l_{o}, \mathbb{S} l_{o}\right) d_{\theta}\left(l_{1}, \mathbb{T} l_{1}\right)}{1+d_{\theta}\left(l_{o}, l_{1}\right)} .
$$

Or

$$
d_{\theta}\left(l_{1}, l_{2}\right) \preceq \lambda_{1} d_{\theta}\left(l_{o}, l_{1}\right)+\lambda_{2} d_{\theta}\left(l_{1}, l_{2}\right)+\frac{\lambda_{3} d_{\theta}\left(l_{o}, l_{1}\right) d_{\theta}\left(l_{1}, l_{2}\right)}{1+d_{\theta}\left(l_{o}, l_{1}\right)},
$$

from which we have

$$
\left|d_{\theta}\left(l_{1}, l_{2}\right)\right| \leq \lambda_{1}\left|d_{\theta}\left(l_{o}, l_{1}\right)\right|+\lambda_{2}\left|d_{\theta}\left(l_{1}, l_{2}\right)\right|+\frac{\lambda_{3}\left|d_{\theta}\left(l_{o}, l_{1}\right)\right|\left|d_{\theta}\left(l_{1}, l_{2}\right)\right|}{1+\left|d_{\theta}\left(l_{0}, l_{1}\right)\right|} .
$$

That is,

$$
\left|d_{\theta}\left(l_{1}, l_{2}\right)\right| \leq \lambda_{1}\left|d_{\theta}\left(l_{o}, l_{1}\right)\right|+\lambda_{2}\left|d_{\theta}\left(l_{1}, l_{2}\right)\right|+\lambda_{3}\left|d_{\theta}\left(l_{1}, l_{2}\right)\right|,
$$

or

$$
\left|d_{\theta}\left(l_{1}, l_{2}\right)\right| \leq \frac{\lambda_{1}}{\left(1-\lambda_{2}-\lambda_{3}\right)}\left|d_{\theta}\left(l_{0}, l_{1}\right)\right| .
$$

Inductively, we develop a sequence $\left\{l_{p}\right\}$ in $G$ such that

$$
\begin{aligned}
\left|d_{\theta}\left(l_{1}, l_{2}\right)\right| & \leq k\left|d_{\theta}\left(l_{0}, l_{1}\right)\right| . \\
\left|d_{\theta}\left(l_{2}, l_{3}\right)\right| & \leq k^{2}\left|d_{\theta}\left(l_{0}, l_{1}\right)\right| . \\
\cdot & \\
\cdot & \cdot \\
\left|d_{\theta}\left(l_{p}, l_{p+1}\right)\right| & \leq k^{p}\left|d_{\theta}\left(l_{o}, l_{1}\right)\right| .
\end{aligned}
$$

Now, by triangular inequality and for $q>p$, we have the following

$$
\begin{aligned}
d_{\theta}\left(l_{p}, l_{q}\right) \preceq \theta\left(l_{p}, l_{q}\right) k^{p} d_{\theta}\left(l_{o}, l_{1}\right)+\theta\left(l_{p}, l_{q}\right) \theta\left(l_{p+1}, l_{q}\right) k^{p+1} d_{\theta}\left(l_{o}, l_{1}\right)+\cdots \\
\\
+\theta\left(l_{p}, l_{q}\right) \theta\left(l_{p+1}, l_{q}\right) \cdots \theta\left(l_{q-2}, l_{q}\right) \theta\left(l_{q-1}, l_{q}\right) k^{q-1} d_{\theta}\left(l_{o}, l_{1}\right) \cdot d_{\theta}\left(l_{p}, l_{q}\right)
\end{aligned}
$$




$$
\begin{aligned}
d_{\theta}\left(l_{p}, l_{q}\right) & \preceq d_{\theta}\left(l_{0}, l_{1}\right)\left[\theta\left(l_{p}, l_{q}\right) k^{p}+\theta\left(l_{p}, l_{q}\right) \theta\left(l_{p+1}, l_{q}\right) k^{p+1}+\cdots\right. \\
& \left.+\theta\left(l_{p}, l_{q}\right) \theta\left(l_{p+1}, l_{q}\right) \ldots \theta\left(l_{q-2}, l_{q}\right) \theta\left(l_{q-1}, m_{p}\right) k^{q-1}\right]
\end{aligned}
$$

Since $\lim _{p, q \rightarrow \infty} \theta\left(l_{p}, l_{q}\right) k<1$, so the series $\sum_{p=1}^{\infty} k^{p} \prod_{i=1}^{p} \theta\left(l_{i}, l_{q}\right)$ converges by ratio test for each $q \in N$. Let

$$
S=\sum_{p=1}^{\infty} k^{p} \prod_{i=1}^{p} \theta\left(l_{i}, l_{q}\right), \quad S_{p}=\sum_{j=1}^{p} k^{j} \prod_{i=1}^{p} \theta\left(l_{i}, l_{q}\right)
$$

Thus, for $q>p$, the above can be written as

$$
d_{\theta}\left(l_{p}, l_{q}\right) \preceq d_{\theta}\left(l_{0}, l_{1}\right)\left[S_{q-1}-S_{P}\right],
$$

or

$$
\left|d_{\theta}\left(l_{p}, l_{q}\right)\right| \leq\left|d_{\theta}\left(l_{0}, l_{1}\right)\right|\left[S_{q-1}-S_{p}\right]
$$

Now, by taking $p \rightarrow \infty$, we get

$$
\left|d_{\theta}\left(l_{p}, l_{q}\right)\right| \rightarrow 0
$$

By Lemma 2.4, we conclude that $\left\{l_{p}\right\}$ is a Cauchy sequence. Since $\mathbb{G}$ is complete, then there exists an element $r$ such that $l_{p} \rightarrow r \in \mathbb{G}$ as $p \rightarrow \infty$.

To show that $r \in \mathbb{S} r$ and $r \in \mathbb{T} r$, from (6.1), we have

$$
\begin{gathered}
\lambda_{1} d_{\theta}\left(l_{2 p}, \mathbb{S} l_{2 p}\right)+\lambda_{2} d_{\theta}(r, \mathbb{T} r)+\frac{\lambda_{3} d_{\theta}\left(l_{2 p}, \mathbb{S} l_{2 p}\right) d_{\theta}(r, \mathbb{T} r)}{1+d_{\theta}\left(l_{2 p}, r\right)} \in s\left(\mathbb{S} l_{2 p}, \mathbb{T} r\right), \\
\lambda_{1} d_{\theta}\left(l_{2 p}, S l_{2 p}\right)+\lambda_{2} d_{\theta}(r, \mathbb{T} r)+\frac{\lambda_{3} d_{\theta}\left(l_{2 p}, \mathbb{S} l_{2 p}\right) d_{\theta}(r, \mathbb{T} r)}{1+d_{\theta}\left(l_{2 p}, r\right)} \in \bigcap_{a^{\prime} \in S l_{2 p}} s\left(a^{\prime}, \mathbb{T} r\right) .
\end{gathered}
$$

Since $l_{2 p+1} \in \mathbb{S} l_{2 p}$, we have

$$
\begin{gathered}
\lambda_{1} d_{\theta}\left(l_{2 p}, S l_{2 p}\right)+\lambda_{2} d_{\theta}(r, \mathbb{T} r)+\frac{\lambda_{3} d_{\theta}\left(l_{2 p}, \mathbb{S} l_{2 p}\right) d_{\theta}(r, \mathbb{T} r)}{1+d_{\theta}\left(l_{2 p}, r\right)} \in s\left(l_{2 p+1}, \mathbb{T} r\right) \\
\lambda_{1} d_{\theta}\left(l_{2 p}, S l_{2 p}\right)+\lambda_{2} d_{\theta}(r, \mathbb{T} r)+\frac{\lambda_{3} d_{\theta}\left(l_{2 p}, \mathbb{S} l_{2 p}\right) d_{\theta}(r, \mathbb{T} r)}{1+d_{\theta}\left(l_{2 p}, r\right)} \in \bigcup_{b^{\prime} \in \mathbb{T} r} s\left(d_{\theta}\left(l_{2 p+1}, b^{\prime}\right) .\right.
\end{gathered}
$$

So, there exist some $r_{p} \in \mathbb{T} r$ such that

$$
\lambda_{1} d_{\theta}\left(l_{2 p}, S l_{2 p}\right)+\lambda_{2} d_{\theta}(r, \mathbb{T} r)+\frac{\lambda_{3} d_{\theta}\left(l_{2 p}, S l_{2 p}\right) d_{\theta}(r, \mathbb{T} r)}{1+d_{\theta}\left(l_{2 p}, r\right)} \in s\left(d_{\theta}\left(l_{2 p+1}, r_{p}\right) .\right.
$$

Using definition 2.5, we have

$$
d_{\theta}\left(l_{2 p+1}, r_{p}\right) \preceq \lambda_{1} d_{\theta}\left(l_{2 p}, \mathbb{S} l_{2 p}\right)+\lambda_{2} d_{\theta}(r, \mathbb{T} r)+\frac{\lambda_{3} d_{\theta}\left(l_{2 p}, \mathbb{S} l_{2 p}\right) d_{\theta}(r, \mathbb{T} r)}{1+d_{\theta}\left(l_{2 p}, r\right)} .
$$

The g.l.b property of $\mathbb{T}$ yields

$$
d_{\theta}\left(l_{2 p+1}, r_{p}\right) \preceq \lambda_{1} d_{\theta}\left(l_{2 p}, l_{2 p+1}\right)+\lambda_{2} d_{\theta}\left(r, r_{p}\right)+\frac{\lambda_{3} d_{\theta}\left(l_{2 p}, l_{2 p+1}\right) d_{\theta}\left(r, r_{p}\right)}{1+d_{\theta}\left(l_{2 p}, r\right)} .
$$

Now,

$$
\begin{array}{r}
d_{\theta}\left(r, r_{p}\right) \preceq \theta\left(r, r_{p}\right)\left[d_{\theta}\left(r, l_{2 p+1}\right)+d_{\theta}\left(l_{2 p+1}, r_{p}\right)\right] \\
d_{\theta}\left(r, r_{p}\right) \preceq \theta\left(r, r_{p}\right) d_{\theta}\left(r, l_{2 p+1}\right)+\theta\left(r, r_{p}\right) \lambda_{1} d_{\theta}\left(l_{2 p}, l_{2 p+1}\right)
\end{array}
$$




$$
+\theta\left(r, r_{p}\right) \lambda_{2} d_{\theta}\left(r, r_{p}\right)+\frac{\theta\left(r, r_{p}\right) \lambda_{3} d_{\theta}\left(l_{2 p}, l_{2 p+1}\right) d_{\theta}\left(r, r_{p}\right)}{1+d_{\theta}\left(l_{2 p}, r\right)}
$$

This implies

$$
\begin{aligned}
\left|d_{\theta}\left(r, r_{p}\right)\right| & \leq \theta\left(r, r_{p}\right)\left|d_{\theta}\left(r, l_{2 p+1}\right)\right|+\theta\left(r, r_{p}\right) \lambda_{1}\left|d_{\theta}\left(l_{2 p}, l_{2 p+1}\right)\right| \\
& +\theta\left(r, r_{p}\right) \lambda_{2}\left|d_{\theta}\left(r, r_{p}\right)\right|+\frac{\theta\left(r, r_{p}\right) \lambda_{3}\left|d_{\theta}\left(l_{2 p}, l_{2 p+1}\right)\right|\left|d_{\theta}\left(r, r_{p}\right)\right|}{1+\left|d_{\theta}\left(l_{2 p}, r\right)\right|} .
\end{aligned}
$$

Taking the limit as $p \rightarrow \infty$, we get $\left|d_{\theta}\left(r, r_{p}\right)\right| \rightarrow 0$.

By Lemma 2.4, we have $r_{p} \rightarrow r$. Since $\mathbb{T}$ is closed, then $r \in \mathbb{T} r$. By similar arguments, we can show that $r \in \mathrm{S} r$. Thus, $\mathrm{S}$ and $\mathbb{T}$ have a common fixed point.

By setting $\mathrm{S}=\mathbb{T}$ in Theorem 4.1, we get

Corollary 4.1. Let $\left(\mathbb{G}, d_{\theta}\right)$ be a complete $C . V$ extended b-metric space, $\theta: \mathbb{G} \times \mathbb{G} \rightarrow[1, \infty)$ be a function and $T: \mathbb{G} \rightarrow$ $C B(\mathbb{G})$ be a multi-valued mapping satisfying the g.l.b property such that

$$
\lambda_{1} d_{\theta}(l, \mathbb{T} l)+\lambda_{2} d_{\theta}(m, \mathbb{T} m)+\frac{\lambda_{3} d_{\theta}(l, \mathbb{T} r) d_{\theta}(m, \mathbb{T} m)}{1+d_{\theta}(l, m)} \in s(\mathbb{T} l, \mathbb{T} m),
$$

for all $l, m \in \mathbb{G}$ and $\lambda_{1}, \lambda_{2}, \lambda_{3}$ are non negative reals with $\lambda_{1}+\lambda_{2}+\lambda_{3}<1$, and assumed that $k\left(1-\lambda_{2}-\lambda_{3}\right)=\lambda_{1}$, where $k \in[0,1)$ is such that for each $l_{o} \in \mathbb{G}, \lim _{p, q \rightarrow \infty} \theta\left(l_{p}, l_{q}\right) k<1$. Then $\mathbb{T}$ has a fixed point.

Theorem 4.2. Let $\left(\mathbb{G}, d_{\theta}\right)$ be a complete $C . V$ extended b-metric space, $\theta: \mathbb{G} \times \mathbb{G} \rightarrow[1, \infty)$ be a function and $\mathbb{T}: \mathbb{G} \rightarrow$ $C B(G)$ be a multi-valued mapping fulfilling the g.l.b property such that

$$
\begin{aligned}
& \lambda_{1} d_{\theta}(l, m)+\lambda_{2} \frac{d_{\theta}(l, \mathbb{T} l) d_{\theta}(m, \mathbb{T} m)}{1+d(l, m)}+\lambda_{3} \frac{\left.d_{\theta}(m, \mathbb{T} l) d_{(} l, \mathbb{T} m\right)}{1+d_{\theta}(l, m)} \\
& +\lambda_{4} \frac{d_{\theta}(l, \mathbb{T} l) d_{\theta}(l, \mathbb{T} m)}{\left.1+d_{(} l, m\right)}+\lambda_{5} \frac{d_{\theta}(m, \mathbb{T} l) d_{\theta}(m, \mathbb{T} m)}{1+d_{\theta}(l, m)} \in s(\mathbb{T} l, \mathbb{T} m),
\end{aligned}
$$

for all $l, m \in \mathbb{G}$ and $\lambda_{1}, \lambda_{2}, \lambda_{3}, \lambda_{4}, \lambda_{5}$ are nonnegative real numbers with $\lambda_{1}+\lambda_{2}+\lambda_{3}$

$+2 \lambda_{4} \theta\left(l_{0}, l_{2}\right)+\lambda_{5}<1$ and assumed that $k\left(1-\lambda_{2}-\lambda_{4} \theta\left(l_{0}, l_{2}\right)\right)=\lambda_{1}+\lambda_{4} \theta\left(l_{0}, l_{2}\right)$ where $k \in[0,1)$ is such that for each $l_{o} \in \mathbb{G}, \lim _{p, q \rightarrow \infty} \theta\left(l_{p}, l_{q}\right) k<1$. Then $\mathbb{T}$ has a fixed point.

Proof. Let $l_{o} \in \mathrm{G}$, then $T l_{o}$ is non-empty, so we can take $l_{1} \in T l_{o}$; thus from (4.3),

$$
\begin{gathered}
\lambda_{1} d_{\theta}\left(l_{o}, l_{1}\right)+\lambda_{2} \frac{d_{\theta}\left(l_{o}, \mathbb{T} l_{o}\right) d_{\theta}\left(l_{1}, \mathbb{T} l_{1}\right)}{1+d\left(l_{o}, l_{1}\right)}+\lambda_{3} \frac{\left.d_{\theta}\left(l_{1}, \mathbb{T} l_{o}\right) d_{(}, l_{o}, \mathbb{T} l_{1}\right)}{1+d_{\theta}\left(l_{0}, l_{1}\right)} \\
+\lambda_{4} \frac{d_{\theta}\left(l_{o}, \mathbb{T} l_{o}\right) d_{\theta}\left(l_{o}, \mathbb{T} l_{1}\right)}{\left.1+d_{(} l_{o}, l_{1}\right)}+\lambda_{5} \frac{\left.d_{\theta}\left(l_{1}, \mathbb{T} l_{o}\right) d_{(} l_{1}, \mathbb{T} l_{1}\right)}{\left.1+d_{(} l_{0}, l_{1}\right)} \in s\left(\mathbb{T} l_{o}, \mathbb{T} l_{1}\right), \\
\lambda_{1} d_{\theta}\left(l_{o}, l_{1}\right)+\lambda_{2} \frac{d_{\theta}\left(l_{o}, \mathbb{T} l_{o}\right) d_{\theta}\left(l_{1}, \mathbb{T} l_{1}\right)}{1+d\left(l_{o}, l_{1}\right)}+\lambda_{3} \frac{\left.d_{\theta}\left(l_{1}, \mathbb{T} l_{o}\right) d_{(} l_{o}, \mathbb{T} l_{1}\right)}{1+d_{\theta}\left(l_{0}, l_{1}\right)} \\
+\lambda_{4} \frac{d_{\theta}\left(l_{o}, \mathbb{T} l_{o}\right) d_{\theta}\left(l_{o}, \mathbb{T} l_{1}\right)}{\left.1+d_{(} l_{o}, l_{1}\right)}+\lambda_{5} \frac{\left.d_{\theta}\left(l_{1}, \mathbb{T} l_{o}\right) d_{(} l_{1}, \mathbb{T} l_{1}\right)}{\left.1+d_{(} l_{o}, l_{1}\right)} \in \bigcap_{a \in \mathbb{T} l_{o}} s\left(a, \mathbb{T} l_{1}\right), \\
\lambda_{1} d_{\theta}\left(l_{o}, l_{1}\right)+\lambda_{2} \frac{d_{\theta}\left(l_{o}, \mathbb{T} l_{o}\right) d_{\theta}\left(l_{1}, \mathbb{T} l_{1}\right)}{1+d\left(l_{o}, l_{1}\right)}+\lambda_{3} \frac{\left.d_{\theta}\left(l_{1}, \mathbb{T} l_{o}\right) d_{(} l_{o}, \mathbb{T} l_{1}\right)}{1+d_{\theta}\left(l_{0}, l_{1}\right)} \\
+\lambda_{4} \frac{d_{\theta}\left(l_{o}, \mathbb{T} l_{o}\right) d_{\theta}\left(l_{o}, \mathbb{T} l_{1}\right)}{\left.1+d_{(} l_{o}, l_{1}\right)}+\lambda_{5} \frac{\left.d_{\theta}\left(l_{1}, \mathbb{T} l_{o}\right) d_{(} l_{1}, \mathbb{T} l_{1}\right)}{\left.1+d_{(} l_{o}, l_{1}\right)} \in s\left(a, \mathbb{T} l_{1}\right) \quad \forall a \in \mathbb{T} l_{o} .
\end{gathered}
$$


Since $l_{1} \in \mathbb{T} l_{0}$, we get

$$
\begin{gathered}
\lambda_{1} d_{\theta}\left(l_{o}, l_{1}\right)+\lambda_{2} \frac{d_{\theta}\left(l_{o}, \mathbb{T} l_{o}\right) d_{\theta}\left(l_{1}, \mathbb{T} l_{1}\right)}{1+d\left(l_{o}, l_{1}\right)}+\lambda_{3} \frac{\left.d_{\theta}\left(l_{1}, \mathbb{T} l_{o}\right) d_{(} l_{o}, \mathbb{T} l_{1}\right)}{1+d_{\theta}\left(l_{0}, l_{1}\right)} \\
+\lambda_{4} \frac{d_{\theta}\left(l_{o}, \mathbb{T} l_{o}\right) d_{\theta}\left(l_{o}, \mathbb{T} l_{1}\right)}{\left.1+d_{(} l_{o}, l_{1}\right)}+\lambda_{5} \frac{d_{\theta}\left(l_{1}, \mathbb{T} l_{o}\right) d_{(}\left(l_{1}, \mathbb{T} l_{1}\right)}{\left.1+d_{(} l_{o}, l_{1}\right)} \in s\left(l_{1}, \mathbb{T} l_{1}\right), \\
\lambda_{1} d_{\theta}\left(l_{o}, l_{1}\right)+\lambda_{2} \frac{d_{\theta}\left(l_{o}, \mathbb{T} l_{o}\right) d_{\theta}\left(l_{1}, \mathbb{T} l_{1}\right)}{1+d\left(l_{o}, l_{1}\right)}+\lambda_{3} \frac{\left.d_{\theta}\left(l_{1}, \mathbb{T} l_{o}\right) d_{(} l_{o}, \mathbb{T} l_{1}\right)}{1+d_{\theta}\left(l_{0}, l_{1}\right)} \\
+\lambda_{4} \frac{d_{\theta}\left(l_{o}, \mathbb{T} l_{o}\right) d_{\theta}\left(l_{o}, \mathbb{T} l_{1}\right)}{\left.1+d_{(} l_{o}, l_{1}\right)}+\lambda_{5} \frac{d_{\theta}\left(l_{1}, \mathbb{T} l_{o}\right) d_{(}\left(l_{1}, \mathbb{T} l_{1}\right)}{\left.1+d_{(} l_{o}, l_{1}\right)} \in \bigcup_{b \in \mathbb{T} x_{1}} s\left(d_{\theta}\left(l_{1}, b\right)\right) .
\end{gathered}
$$

Thus, there exists some $l_{2} \in \mathbb{T} l_{1}$ such that

$$
\begin{aligned}
& \lambda_{1} d_{\theta}\left(l_{o}, l_{1}\right)+\lambda_{2} \frac{d_{\theta}\left(l_{o}, \mathbb{T} l_{o}\right) d_{\theta}\left(l_{1}, \mathbb{T} l_{1}\right)}{1+d\left(l_{0}, l_{1}\right)}+\lambda_{3} \frac{\left.d_{\theta}\left(l_{1}, \mathbb{T} l_{o}\right) d_{(} l_{o}, \mathbb{T} l_{1}\right)}{1+d_{\theta}\left(l_{0}, l_{1}\right)} \\
& +\lambda_{4} \frac{d_{\theta}\left(l_{o}, \mathbb{T} l_{o}\right) d_{\theta}\left(l_{o}, \mathbb{T} l_{1}\right)}{\left.1+d_{(} l_{o}, l_{1}\right)}+\lambda_{5} \frac{\left.d_{\theta}\left(l_{1}, \mathbb{T} l_{o}\right) d_{(} l_{1}, \mathbb{T} l_{1}\right)}{\left.1+d_{(} l_{o}, l_{1}\right)} \in s\left(d_{\theta}\left(l_{1}, l_{2}\right)\right) .
\end{aligned}
$$

By definition 2.5

$$
\begin{aligned}
d_{\theta}\left(l_{1}, l_{2}\right) & \preceq \lambda_{1} d_{\theta}\left(l_{o}, l_{1}\right)+\lambda_{2} \frac{d_{\theta}\left(l_{o}, \mathbb{T} l_{o}\right) d_{\theta}\left(l_{1}, \mathbb{T} l_{1}\right)}{1+d\left(l_{0}, l_{1}\right)}+\lambda_{3} \frac{d_{\theta}\left(l_{1}, \mathbb{T} l_{o}\right) d_{(}\left(l_{o}, \mathbb{T} l_{1}\right)}{1+d_{\theta}\left(l_{0}, l_{1}\right)} \\
& +\lambda_{4} \frac{d_{\theta}\left(l_{o}, \mathbb{T} l_{o}\right) d_{\theta}\left(l_{o}, \mathbb{T} l_{1}\right)}{\left.1+d_{(} l_{o}, l_{1}\right)}+\lambda_{5} \frac{\left.d_{\theta}\left(l_{1}, \mathbb{T} l_{o}\right) d_{(} l_{1}, \mathbb{T} l_{1}\right)}{\left.1+d_{(} l_{o}, l_{1}\right)} .
\end{aligned}
$$

Using the g.l.b property of $\mathbb{T}$, we get

$$
\begin{aligned}
d_{\theta}\left(l_{1}, l_{2}\right) & \preceq \lambda_{1} d_{\theta}\left(l_{0}, l_{1}\right)+\lambda_{2} \frac{d_{\theta}\left(l_{0}, l_{1}\right) d_{\theta}\left(l_{1}, l_{2}\right)}{1+d\left(l_{0}, l_{1}\right)}+\lambda_{3} \frac{\left.d_{\theta}\left(l_{1}, l_{1}\right) d_{(} l_{o}, l_{2}\right)}{1+d_{\theta}\left(l_{0}, l_{1}\right)} \\
& +\lambda_{4} \frac{d_{\theta}\left(l_{0}, l_{1}\right) d_{\theta}\left(l_{o}, l_{2}\right)}{\left.1+d_{(} l_{0}, l_{1}\right)}+\lambda_{5} \frac{\left.d_{\theta}\left(l_{1}, l_{1}\right) d_{(} l_{1}, l_{2}\right)}{\left.1+d_{(} l_{0}, l_{1}\right)}
\end{aligned}
$$

The above inequality gives

$$
\left|d_{\theta}\left(l_{1}, l_{2}\right)\right| \leq \lambda_{1}\left|d_{\theta}\left(l_{o}, l_{1}\right)\right|+\lambda_{2}\left|d_{\theta}\left(l_{1}, l_{2}\right)\right|+\lambda_{4}\left|d_{\theta}\left(l_{o}, l_{2}\right)\right| .
$$

By using the triangular inequality, we get the following

$$
\begin{aligned}
\left|d_{\theta}\left(l_{1}, l_{2}\right)\right| & \leq \lambda_{1}\left|d_{\theta}\left(l_{0}, l_{1}\right)\right|+\lambda_{2}\left|d_{\theta}\left(l_{1}, l_{2}\right)\right| \\
& +\theta\left(l_{0}, l_{2}\right) \lambda_{4}\left|d_{\theta}\left(l_{0}, l_{1}\right)\right|+\theta\left(l_{0}, l_{2}\right) \lambda_{4}\left|d_{\theta}\left(l_{1}, l_{2}\right)\right| .
\end{aligned}
$$

Thus,

$$
\left|d_{\theta}\left(l_{1}, l_{2}\right)\right| \leq \frac{\lambda_{1}+\theta\left(l_{0}, l_{2}\right) \lambda_{4}}{1-\lambda_{2}-\theta\left(l_{0}, l_{2}\right) \lambda_{4}}\left|d_{\theta}\left(l_{o}, l_{1}\right)\right| .
$$

Inductively, we develop a sequence $\left\{l_{p}\right\}$ in $G$ such that

$$
\begin{aligned}
& \left|d_{\theta}\left(l_{1}, l_{2}\right)\right| \leq k\left|d_{\theta}\left(l_{0}, l_{1}\right)\right| \\
& \left|d_{\theta}\left(l_{2}, l_{3}\right)\right| \leq k^{2}\left|d_{\theta}\left(l_{0}, l_{1}\right)\right|
\end{aligned}
$$




$$
\left|d_{\theta}\left(l_{p}, l_{p+1}\right)\right| \leq k^{p}\left|d_{\theta}\left(l_{0}, l_{1}\right)\right| .
$$

Now, by triangular inequality and for $q>p$, we have the following

$$
\begin{aligned}
d_{\theta}\left(l_{p}, l_{q}\right) & \preceq \theta\left(l_{p}, l_{q}\right) k^{p} d_{\theta}\left(l_{o}, l_{1}\right)+\theta\left(l_{p}, l_{q}\right) \theta\left(l_{p+1}, l_{q}\right) k^{p+1} d_{\theta}\left(l_{o}, l_{1}\right)+\cdots \\
& +\theta\left(l_{p}, l_{q}\right) \theta\left(l_{p+1}, l_{q}\right) \cdots \theta\left(l_{q-2}, l_{q}\right) \theta\left(l_{q-1}, l_{q}\right) k^{q-1} d_{\theta}\left(l_{o}, l_{1}\right) \cdot d_{\theta}\left(l_{p}, l_{q}\right) \\
d_{\theta}\left(l_{p}, l_{q}\right) & \preceq d_{\theta}\left(l_{0}, l_{1}\right)\left[\theta\left(l_{p}, l_{q}\right) k^{p}+\theta\left(l_{p}, l_{q}\right) \theta\left(l_{p+1}, l_{q}\right) k^{p+1}+\cdots\right. \\
& \left.+\theta\left(l_{p}, l_{q}\right) \theta\left(l_{p+1}, l_{q}\right) \ldots \theta\left(l_{q-2}, l_{q}\right) \theta\left(l_{q-1}, m_{p}\right) k^{q-1}\right] .
\end{aligned}
$$

Since $\lim _{p, q \rightarrow \infty} \theta\left(l_{p}, l_{q}\right) k<1$, so the series $\sum_{p=1}^{\infty} k^{p} \prod_{i=1}^{p} \theta\left(l_{i}, l_{q}\right)$ converges by ratio test for each $q \in N$. Let

$$
S=\sum_{p=1}^{\infty} k^{p} \prod_{i=1}^{p} \theta\left(l_{i}, l_{q}\right), \quad S_{p}=\sum_{j=1}^{p} k^{j} \prod_{i=1}^{p} \theta\left(l_{i}, l_{q}\right)
$$

Thus, for $q>p$, the above can be written as

$$
\begin{aligned}
d_{\theta}\left(l_{p}, l_{q}\right) & \preceq d_{\theta}\left(l_{o}, l_{1}\right)\left[S_{q-1}-S_{P}\right] \\
\left|d_{\theta}\left(l_{p}, l_{q}\right)\right| & \leq\left|d_{\theta}\left(l_{o}, l_{1}\right)\right|\left[S_{q-1}-S_{p}\right] .
\end{aligned}
$$

Now, by taking $p \rightarrow \infty$, we get

$$
\left|d_{\theta}\left(l_{p}, l_{q}\right)\right| \rightarrow 0 .
$$

By Lemma 2.4, we conclude that $\left\{l_{p}\right\}$ is a Cauchy sequence. By the completeness of $\mathbb{G}$, there exists an element $r$ such that $l_{p} \rightarrow r \in \mathrm{G}$ as $p \rightarrow \infty$.

We show $r \in \mathbb{T} r$. From (6.3), we have

$$
\begin{aligned}
& \lambda_{1} d_{\theta}\left(l_{2 p}, r\right)+\lambda_{2} \frac{d_{\theta}\left(l_{2 p}, \mathbb{T} l_{2 p}\right) d_{\theta}(r, \mathbb{T} r)}{1+d\left(l_{2 p}, r\right)}+\lambda_{3} \frac{\left.d_{\theta}\left(r, \mathbb{T} l_{2 p}\right) d_{(} l_{2 p}, \mathbb{T} r\right)}{1+d_{\theta}\left(l_{2 p}, r\right)} \\
& +\lambda_{4} \frac{d_{\theta}\left(l_{2 p}, \mathbb{T} l_{2 p}\right) d_{\theta}\left(l_{2 p}, \mathbb{T} r\right)}{\left.1+d_{(} l_{2 p}, r\right)}+\lambda_{5} \frac{d_{\theta}\left(r, \mathbb{T} l_{2 p}\right) d_{\theta}(r, \mathbb{T} r)}{1+d_{\theta}\left(l_{2 p}, r\right)} \in s\left(\mathbb{T} l_{2 p}, \mathbb{T} r\right), \\
& \lambda_{1} d_{\theta}\left(l_{2 p}, r\right)+\lambda_{2} \frac{d_{\theta}\left(l_{2 p}, \mathbb{T} l_{2 p}\right) d_{\theta}(r, \mathbb{T} r)}{1+d\left(l_{2 p}, r\right)}+\lambda_{3} \frac{\left.d_{\theta}\left(r, \mathbb{T} l_{2 p}\right) d_{(} l_{2 p}, \mathbb{T} r\right)}{1+d_{\theta}\left(l_{2 p}, r\right)} \\
& +\lambda_{4} \frac{d_{\theta}\left(l_{2 p}, \mathbb{T} l_{2 p}\right) d_{\theta}\left(l_{2 p}, \mathbb{T} r\right)}{\left.1+d_{(} l_{2 p}, r\right)}+\lambda_{5} \frac{d_{\theta}\left(r, \mathbb{T} l_{2 p}\right) d_{\theta}(r, \mathbb{T} r)}{1+d_{\theta}\left(l_{2 p}, r\right)} \in \bigcap_{a \in \mathbb{T} l_{2 p}} s(a, \mathbb{T} r) .
\end{aligned}
$$

Since $l_{2 p+1} \in \mathbb{T} l_{2 p}$, we get

$$
\begin{gathered}
\lambda_{1} d_{\theta}\left(l_{2 p}, r\right)+\lambda_{2} \frac{d_{\theta}\left(l_{2 p}, \mathbb{T} l_{2 p}\right) d_{\theta}(r, \mathbb{T} r)}{1+d\left(l_{2 p}, r\right)}+\lambda_{3} \frac{\left.d_{\theta}\left(r, \mathbb{T} l_{2 p}\right) d_{(} l_{2 p}, \mathbb{T} r\right)}{1+d_{\theta}\left(l_{2 p}, r\right)} \\
+\lambda_{4} \frac{d_{\theta}\left(l_{2 p}, \mathbb{T} l_{2 p}\right) d_{\theta}\left(l_{2 p}, \mathbb{T} r\right)}{\left.1+d_{(} l_{2 p}, r\right)}+\lambda_{5} \frac{d_{\theta}\left(r, \mathbb{T} l_{2 p}\right) d_{\theta}(r, \mathbb{T} r)}{1+d_{\theta}\left(l_{2 p}, r\right)} \in s\left(l_{2 p+1}, \mathbb{T} r\right), \\
\lambda_{1} d_{\theta}\left(l_{2 p}, r\right)+\lambda_{2} \frac{d_{\theta}\left(l_{2 p}, \mathbb{T} l_{2 p}\right) d_{\theta}(r, \mathbb{T} r)}{1+d\left(l_{2 p}, r\right)}+\lambda_{3} \frac{\left.d_{\theta}\left(r, \mathbb{T} l_{2 p}\right) d_{(} l_{2 p}, \mathbb{T} r\right)}{1+d_{\theta}\left(l_{2 p}, r\right)} \\
+\lambda_{4} \frac{d_{\theta}\left(l_{2 p}, \mathbb{T} l_{2 p}\right) d_{\theta}\left(l_{2 p}, \mathbb{T} r\right)}{\left.1+d_{(} l_{2 p}, r\right)}+\lambda_{5} \frac{d_{\theta}\left(r, \mathbb{T} l_{2 p}\right) d_{\theta}(r, \mathbb{T} r)}{1+d_{\theta}\left(l_{2 p}, r\right)} \in \bigcup_{b \in \mathbb{T} r} s\left(d_{\theta}\left(l_{2 p+1}, b\right)\right) .
\end{gathered}
$$


So, there exists some $r_{p} \in \mathbb{T} r$ such that

$$
\begin{aligned}
& \lambda_{1} d_{\theta}\left(l_{2 p}, r\right)+\lambda_{2} \frac{d_{\theta}\left(l_{2 p}, \mathbb{T} l_{2 p}\right) d_{\theta}(r, \mathbb{T} r)}{1+d\left(l_{2 p}, r\right)}+\lambda_{3} \frac{\left.d_{\theta}\left(r, \mathbb{T} l_{2 p}\right) d_{(} l_{2 p}, \mathbb{T} r\right)}{1+d_{\theta}\left(l_{2 p}, r\right)} \\
& +\lambda_{4} \frac{d_{\theta}\left(l_{2 p}, \mathbb{T} l_{2 p}\right) d_{\theta}\left(l_{2 p}, \mathbb{T} r\right)}{\left.1+d_{(} l_{2 p}, r\right)}+\lambda_{5} \frac{d_{\theta}\left(r, \mathbb{T} l_{2 p}\right) d_{\theta}(r, \mathbb{T} r)}{1+d_{\theta}\left(l_{2 p}, r\right)} \in s\left(d_{\theta}\left(l_{2 p+1}, r_{p}\right)\right) .
\end{aligned}
$$

Therefore, by definition (2.5), we have

$$
\begin{aligned}
d_{\theta}\left(l_{2 p+1}, r_{p}\right) \preceq \lambda_{1} d_{\theta}\left(l_{2 p}, r\right)+\lambda_{2} \frac{d_{\theta}\left(l_{2 p}, \mathbb{T} l_{2 p}\right) d_{\theta}(r, \mathbb{T} r)}{1+d\left(l_{2 p}, r\right)}+\lambda_{3} \frac{\left.d_{\theta}\left(r, \mathbb{T} l_{2 p}\right) d_{(} l_{2 p}, \mathbb{T} r\right)}{1+d_{\theta}\left(l_{2 p}, r\right)} \\
+\lambda_{4} \frac{d_{\theta}\left(l_{2 p}, \mathbb{T} l_{2 p}\right) d_{\theta}\left(l_{2 p}, \mathbb{T} r\right)}{\left.1+d_{(} l_{2 p}, r\right)}+\lambda_{5} \frac{d_{\theta}\left(r, \mathbb{T} l_{2 p}\right) d_{\theta}(r, \mathbb{T} r)}{1+d_{\theta}\left(l_{2 p}, r\right)} .
\end{aligned}
$$

Using the g.1.b property of $\mathbb{T}$, gives

$$
\begin{aligned}
d_{\theta}\left(l_{2 p+1}, r_{p}\right) \preceq \lambda_{1} d_{\theta}\left(l_{2 p}, r\right)+\lambda_{2} \frac{d_{\theta}\left(l_{2 p}, l_{2 p+1}\right) d_{\theta}\left(r, r_{p}\right)}{1+d\left(l_{2 p}, r\right)}+\lambda_{3} \frac{d_{\theta}\left(r, l_{2 p+1}\right) d_{(}\left(l_{2 p}, r_{P}\right)}{1+d_{\theta}\left(l_{2 p}, r\right)} \\
+\lambda_{4} \frac{d_{\theta}\left(l_{2 p}, l_{2 p+1}\right) d_{\theta}\left(l_{2 p}, r_{p}\right)}{\left.1+d_{(} l_{2 p}, r\right)}+\lambda_{5} \frac{d_{\theta}\left(r, l_{2 p+1}\right) d_{\theta}\left(r, r_{p}\right)}{1+d_{\theta}\left(l_{2 p}, r\right)} .
\end{aligned}
$$

Now, we have

$$
d_{\theta}\left(r, r_{p}\right) \preceq \theta\left(r, r_{p}\right)\left[d_{\theta}\left(r, l_{2 p+1}\right)+d_{\theta}\left(l_{2 p+1}, r_{p}\right)\right] .
$$

Thus,

$$
\begin{aligned}
d_{\theta}\left(r, r_{p}\right) & \preceq \theta\left(r, r_{p}\right) d_{\theta}\left(r, l_{2 p+1}\right)+\theta\left(r, r_{p}\right) \lambda_{1} d_{\theta}\left(l_{2 p}, r\right)+\theta\left(r, r_{p}\right) \lambda_{2} \frac{d_{\theta}\left(l_{2 p}, l_{2 p+1}\right) d_{\theta}\left(r, r_{p}\right)}{1+d\left(l_{2 p}, r\right)} \\
& +\theta\left(r, r_{p}\right) \lambda_{3} \frac{\left.d_{\theta}\left(r, l_{2 p+1}\right) d_{(} l_{2 p}, r_{P}\right)}{1+d_{\theta}\left(l_{2 p}, r\right)}+\theta\left(r, r_{p}\right) \lambda_{4} \frac{d_{\theta}\left(l_{2 p}, l_{2 p+1}\right) d_{\theta}\left(l_{2 p}, r_{p}\right)}{\left.1+d_{(} l_{2 p}, r\right)} \\
& +\theta\left(r, r_{p}\right) \lambda_{5} \frac{d_{\theta}\left(r, l_{2 p+1}\right) d_{\theta}\left(r, r_{p}\right)}{1+d_{\theta}\left(l_{2 p}, r\right)} .
\end{aligned}
$$

Which implies

$$
\begin{aligned}
\left|d_{\theta}\left(r, r_{p}\right)\right| & \leq \theta\left(r, r_{p}\right)\left|d_{\theta}\left(r, l_{2 p+1}\right)\right|+\theta\left(r, r_{p}\right) \lambda_{1}\left|d_{\theta}\left(l_{2 p}, r\right)\right|+\theta\left(r, r_{p}\right) \lambda_{2}\left|\frac{d_{\theta}\left(l_{2 p}, l_{2 p+1}\right) d_{\theta}\left(r, r_{p}\right)}{1+d\left(l_{2 p}, r\right)}\right| \\
& +\theta\left(r, r_{p}\right) \lambda_{3}\left|\frac{\left.d_{\theta}\left(r, l_{2 p+1}\right) d_{(} l_{2 p}, r_{P}\right)}{1+d_{\theta}\left(l_{2 p}, r\right)}\right|+\theta\left(r, r_{p}\right) \lambda_{4}\left|\frac{d_{\theta}\left(l_{2 p}, l_{2 p+1}\right) d_{\theta}\left(l_{2 p}, r_{p}\right)}{\left.1+d_{(} l_{2 p}, r\right)}\right| \\
& +\theta\left(r, r_{p}\right) \lambda_{5}\left|\frac{d_{\theta}\left(r, l_{2 p+1}\right) d_{\theta}\left(r, r_{p}\right)}{1+d_{\theta}\left(l_{2 p}, r\right)}\right| .
\end{aligned}
$$

Taking limit as $p \rightarrow \infty$, we get

$$
\left|d_{\theta}\left(r, r_{p}\right)\right| \rightarrow 0
$$

By Lemma 2.4, $r_{p} \rightarrow r$ as $p \rightarrow \infty$, Also, since $\mathbb{T} r$ is closed , then $r \in \mathbb{T} r$. Thus $\mathbb{T}$ has a fixed point. 
Theorem 4.3. Let $\left(\mathbb{G}, d_{\theta}\right)$ be a complete $C . V$ extended b-metric space, $\theta: \mathbb{G} \times \mathbb{G} \rightarrow[1, \infty)$ be a function and let $\mathbb{S}, \mathbb{T}$ : $\mathbb{G} \rightarrow C B(G)$ be a pair of multi-valued mappings fulfilling the g.l.b property such that

$$
\begin{aligned}
& \lambda_{1} d_{\theta}(l, m)+\lambda_{2} \frac{d_{\theta}(l, \mathbb{S} l) d_{\theta}(m, \mathbb{T} m)}{1+d(l, m)}+\lambda_{3} \frac{\left.d_{\theta}(m, \mathbb{S} l) d_{(} l, \mathbb{T} m\right)}{1+d_{\theta}(l, m)} \\
& +\lambda_{4} \frac{d_{\theta}(l, \mathbb{S} l) d_{\theta}(l, \mathbb{T} m)}{\left.1+d_{(} l, m\right)}+\lambda_{5} \frac{d_{\theta}(m, \mathbb{S} l) d_{\theta}(m, \mathbb{T} m)}{1+d_{\theta}(l, m)} \in s(\mathbb{S} l, \mathbb{T} m),
\end{aligned}
$$

for all $l, m \in \mathbb{G}$ and $\lambda_{1}, \lambda_{2}, \lambda_{3}, \lambda_{4}, \lambda_{5}$, are nonnegative real numbers with $\lambda_{1}+\lambda_{2}+\lambda_{3}$ $+2 \theta \lambda_{4}+\lambda_{5}<1$ and assume $k\left(1-\lambda_{2}-\theta \lambda_{4}\right)=1+\theta \lambda_{4}$ where $k \in[0,1)$ is such that for each $l_{o} \in \mathbb{G}, \lim _{p, q \rightarrow \infty} \theta\left(l_{p}, l_{q}\right) k<1$. Then $\mathbb{S}$ and $\mathbb{T}$ have common fixed point.

Proof. Let $l_{o} \in \mathbb{G}$, then $T l_{o}$ is non-empty, so we can take $l_{1} \in T l_{0}$. Thus, from (4.4),

$$
\begin{aligned}
& \lambda_{1} d_{\theta}\left(l_{o}, l_{1}\right)+\lambda_{2} \frac{d_{\theta}\left(l_{o}, S l_{o}\right) d_{\theta}\left(l_{1}, \mathbb{T} l_{1}\right)}{1+d\left(l_{o}, l_{1}\right)}+\lambda_{3} \frac{\left.d_{\theta}\left(l_{1}, S l_{o}\right) d_{(} l_{o}, \mathbb{T} l_{1}\right)}{1+d_{\theta}\left(l_{0}, l_{1}\right)} \\
& +\lambda_{4} \frac{d_{\theta}\left(l_{o}, S l_{o}\right) d_{\theta}\left(l_{o}, \mathbb{T} l_{1}\right)}{\left.1+d_{(} l_{o}, l_{1}\right)}+\lambda_{5} \frac{\left.d_{\theta}\left(l_{1}, S l_{o}\right) d_{(} l_{1}, \mathbb{T} l_{1}\right)}{\left.1+d_{(} l_{o}, l_{1}\right)} \in s\left(S l_{o}, \mathbb{T} l_{1}\right) .
\end{aligned}
$$

This implies

$$
\begin{gathered}
\lambda_{1} d_{\theta}\left(l_{o}, l_{1}\right)+\lambda_{2} \frac{d_{\theta}\left(l_{o}, S l_{o}\right) d_{\theta}\left(l_{1}, \mathbb{T} l_{1}\right)}{1+d\left(l_{o}, l_{1}\right)}+\lambda_{3} \frac{\left.d_{\theta}\left(l_{1}, S l_{o}\right) d_{(} l_{o}, \mathbb{T} l_{1}\right)}{1+d_{\theta}\left(l_{0}, l_{1}\right)} \\
+\lambda_{4} \frac{d_{\theta}\left(l_{o}, S l_{o}\right) d_{\theta}\left(l_{o}, \mathbb{T} l_{1}\right)}{1+d_{(}\left(l_{o}, l_{1}\right)}+\lambda_{5} \frac{d_{\theta}\left(l_{1}, S l_{o}\right) d_{(}\left(l_{1}, \mathbb{T} l_{1}\right)}{\left.1+d_{(} l_{o}, l_{1}\right)} \in \bigcap_{a \in S l_{o}} s\left(a, \mathbb{T} l_{1}\right), \\
\lambda_{1} d_{\theta}\left(l_{o}, l_{1}\right)+\lambda_{2} \frac{d_{\theta}\left(l_{o}, S l_{o}\right) d_{\theta}\left(l_{1}, \mathbb{T} l_{1}\right)}{1+d\left(l_{o}, l_{1}\right)}+\lambda_{3} \frac{\left.d_{\theta}\left(l_{1}, S l_{o}\right) d_{(} l_{o}, \mathbb{T} l_{1}\right)}{1+d_{\theta}\left(l_{0}, l_{1}\right)} \\
+\lambda_{4} \frac{d_{\theta}\left(l_{o}, S l_{o}\right) d_{\theta}\left(l_{o}, \mathbb{T} l_{1}\right)}{\left.1+d_{(} l_{o}, l_{1}\right)}+\lambda_{5} \frac{\left.d_{\theta}\left(l_{1}, S l_{o}\right) d_{(} l_{1}, \mathbb{T} l_{1}\right)}{\left.1+d_{(} l_{o}, l_{1}\right)} \in s\left(a, S l_{1}\right), \quad \forall a \in \mathbb{S} l_{o} .
\end{gathered}
$$

Since $l_{1} \in \mathbb{S} l_{o}$, we get

$$
\begin{aligned}
& \lambda_{1} d_{\theta}\left(l_{o}, l_{1}\right)+\lambda_{2} \frac{d_{\theta}\left(l_{o}, S l_{o}\right) d_{\theta}\left(l_{1}, \mathbb{T} l_{1}\right)}{1+d\left(l_{o}, l_{1}\right)}+\lambda_{3} \frac{\left.d_{\theta}\left(l_{1}, S l_{o}\right) d_{(} l_{o}, \mathbb{T} l_{1}\right)}{1+d_{\theta}\left(l_{0}, l_{1}\right)} \\
& +\lambda_{4} \frac{d_{\theta}\left(l_{o}, S l_{o}\right) d_{\theta}\left(l_{o}, \mathbb{T} l_{1}\right)}{\left.1+d_{(} l_{0}, l_{1}\right)}+\lambda_{5} \frac{\left.d_{\theta}\left(l_{1}, S l_{o}\right) d_{(} l_{1}, S l_{1}\right)}{\left.1+d_{(} l_{o}, l_{1}\right)} \in s\left(l_{1}, \mathbb{T} l_{1}\right), \\
& \lambda_{1} d_{\theta}\left(l_{0}, l_{1}\right)+\lambda_{2} \frac{d_{\theta}\left(l_{o}, S l_{0}\right) d_{\theta}\left(l_{1}, \mathbb{T} l_{1}\right)}{1+d\left(l_{0}, l_{1}\right)}+\lambda_{3} \frac{\left.d_{\theta}\left(l_{1}, S l_{o}\right) d_{(} l_{o}, \mathbb{T} l_{1}\right)}{1+d_{\theta}\left(l_{0}, l_{1}\right)} \\
& +\lambda_{4} \frac{d_{\theta}\left(l_{o}, S l_{o}\right) d_{\theta}\left(l_{o}, \mathbb{T} l_{1}\right)}{\left.1+d_{(} l_{o}, l_{1}\right)}+\lambda_{5} \frac{\left.d_{\theta}\left(l_{1}, S l_{o}\right) d_{(} l_{1}, \mathbb{T} l_{1}\right)}{\left.1+d_{(} l_{0}, l_{1}\right)} \in \bigcup_{b \in \mathbb{T} x_{1}} s\left(d_{\theta}\left(l_{1}, b\right)\right) .
\end{aligned}
$$

Thus, there exists some $l_{2} \in \mathbb{T} l_{1}$ such that

$$
\begin{aligned}
& \lambda_{1} d_{\theta}\left(l_{o}, l_{1}\right)+\lambda_{2} \frac{d_{\theta}\left(l_{o}, S l_{o}\right) d_{\theta}\left(l_{1}, \mathbb{T} l_{1}\right)}{1+d\left(l_{o}, l_{1}\right)}+\lambda_{3} \frac{\left.d_{\theta}\left(l_{1}, S l_{o}\right) d_{(} l_{o}, \mathbb{T} l_{1}\right)}{1+d_{\theta}\left(l_{0}, l_{1}\right)} \\
& +\lambda_{4} \frac{d_{\theta}\left(l_{o}, S l_{o}\right) d_{\theta}\left(l_{o}, \mathbb{T} l_{1}\right)}{\left.1+d_{(} l_{o}, l_{1}\right)}+\lambda_{5} \frac{\left.d_{\theta}\left(l_{1}, S l_{o}\right) d_{(} l_{1}, \mathbb{T} l_{1}\right)}{\left.1+d_{(} l_{o}, l_{1}\right)} \in s\left(d_{\theta}\left(l_{1}, l_{2}\right)\right) .
\end{aligned}
$$


By definition 2.5, we have

$$
\begin{aligned}
d_{\theta}\left(l_{1}, l_{2}\right) & \preceq \lambda_{1} d_{\theta}\left(l_{o}, l_{1}\right)+\lambda_{2} \frac{d_{\theta}\left(l_{o}, S l_{o}\right) d_{\theta}\left(l_{1}, \mathbb{T} l_{1}\right)}{1+d\left(l_{o}, l_{1}\right)}+\lambda_{3} \frac{\left.d_{\theta}\left(l_{1}, S l_{o}\right) d_{(} l_{o}, \mathbb{T} l_{1}\right)}{1+d_{\theta}\left(l_{0}, l_{1}\right)} \\
& +\lambda_{4} \frac{d_{\theta}\left(l_{o}, S l_{o}\right) d_{\theta}\left(l_{o}, \mathbb{T} l_{1}\right)}{\left.1+d_{(} l_{o}, l_{1}\right)}+\lambda_{5} \frac{\left.d_{\theta}\left(l_{1}, S l_{o}\right) d_{(} l_{1}, \mathbb{T} l_{1}\right)}{\left.1+d_{(} l_{o}, l_{1}\right)} .
\end{aligned}
$$

Using the g.l.b property of $\mathbb{S}$ and $\mathbb{T}$, gives

$$
\begin{aligned}
d_{\theta}\left(l_{1}, l_{2}\right) & \preceq \lambda_{1} d_{\theta}\left(l_{0}, l_{1}\right)+\lambda_{2} \frac{d_{\theta}\left(l_{0}, l_{1}\right) d_{\theta}\left(l_{1}, l_{2}\right)}{1+d\left(l_{0}, l_{1}\right)}+\lambda_{3} \frac{\left.d_{\theta}\left(l_{1}, l_{1}\right) d_{(} l_{0}, l_{2}\right)}{1+d_{\theta}\left(l_{0}, l_{1}\right)} \\
& +\lambda_{4} \frac{d_{\theta}\left(l_{0}, l_{1}\right) d_{\theta}\left(l_{o}, l_{2}\right)}{\left.1+d_{(} l_{0}, l_{1}\right)}+\lambda_{5} \frac{\left.d_{\theta}\left(l_{1}, l_{1}\right) d_{(} l_{1}, l_{2}\right)}{\left.1+d_{(} l_{0}, l_{1}\right)}
\end{aligned}
$$

from which we have

$$
\left|d_{\theta}\left(l_{1}, l_{2}\right)\right| \leq \lambda_{1}\left|d_{\theta}\left(l_{o}, l_{1}\right)\right|+\lambda_{2}\left|d_{\theta}\left(l_{1}, l_{2}\right)\right|+\lambda_{4}\left|d_{\theta}\left(l_{o}, l_{2}\right)\right|
$$

Using the triangular inequality, we get

$$
\begin{aligned}
\left|d_{\theta}\left(l_{1}, l_{2}\right)\right| & \leq \lambda_{1}\left|d_{\theta}\left(l_{o}, l_{1}\right)\right|+\lambda_{2}\left|d_{\theta}\left(l_{1}, l_{2}\right)\right| \\
& +\theta\left(l_{0}, l_{2}\right) \lambda_{4}\left|d_{\theta}\left(l_{0}, l_{1}\right)\right|+\theta\left(l_{o}, l_{2}\right) \lambda_{4}\left|d_{\theta}\left(l_{1}, l_{2}\right)\right| .
\end{aligned}
$$

That is,

$$
\left|d_{\theta}\left(l_{1}, l_{2}\right)\right| \leq \frac{\left(\lambda_{1}+\theta\left(l_{o}, l_{2}\right) \lambda_{4}\right)}{\left(1-\lambda_{2}-\theta\left(l_{o}, l_{2}\right) \lambda_{4}\right)}\left|d_{\theta}\left(l_{o}, l_{1}\right)\right|
$$

Thus,

$$
\left|d_{\theta}\left(l_{1}, l_{2}\right)\right| \leq k\left|d_{\theta}\left(l_{0}, l_{1}\right)\right| .
$$

Inductively, we develop a sequence $l_{p}$ in $G$ such that

$$
\begin{aligned}
\left|d_{\theta}\left(l_{1}, l_{2}\right)\right| & \leq k\left|d_{\theta}\left(l_{0}, l_{1}\right)\right| \\
\left|d_{\theta}\left(l_{2}, l_{3}\right)\right| & \leq k^{2}\left|d_{\theta}\left(l_{0}, l_{1}\right)\right| \\
\cdot & \cdot \\
\cdot & \\
\left|d_{\theta}\left(l_{p}, l_{p+1}\right)\right| & \leq k^{p}\left|d_{\theta}\left(l_{o}, l_{1}\right)\right| .
\end{aligned}
$$

By triangular inequality and for $q>p$, we have

$$
\begin{aligned}
d_{\theta}\left(l_{p}, l_{q}\right) & \preceq \theta\left(l_{p}, l_{q}\right) k^{p} d_{\theta}\left(l_{o}, l_{1}\right)+\theta\left(l_{p}, l_{q}\right) \theta\left(l_{p+1}, l_{q}\right) k^{p+1} d_{\theta}\left(l_{o}, l_{1}\right)+\cdots \\
& +\theta\left(l_{p}, l_{q}\right) \theta\left(l_{p+1}, l_{q}\right) \cdots \theta\left(l_{q-2}, l_{q}\right) \theta\left(l_{q-1}, l_{q}\right) k^{q-1} d_{\theta}\left(l_{o}, l_{1}\right) . d_{\theta}\left(l_{p}, l_{q}\right) \\
d_{\theta}\left(l_{p}, l_{q}\right) & \preceq d_{\theta}\left(l_{0}, l_{1}\right)\left[\theta\left(l_{p}, l_{q}\right) k^{p}+\theta\left(l_{p}, l_{q}\right) \theta\left(l_{p+1}, l_{q}\right) k^{p+1}+\cdots\right. \\
& \left.+\theta\left(l_{p}, l_{q}\right) \theta\left(l_{p+1}, l_{q}\right) \ldots \theta\left(l_{q-2}, l_{q}\right) \theta\left(l_{q-1}, m_{p}\right) k^{q-1}\right]
\end{aligned}
$$

Since $\lim _{p, q \rightarrow \infty} \theta\left(l_{p}, l_{q}\right) k<1$, so the series $\sum_{p=1}^{\infty} k^{p} \prod_{i=1}^{p} \theta\left(l_{i}, l_{q}\right)$ converges by ratio test for each $q \in N$. Take

$$
S=\sum_{p=1}^{\infty} k^{p} \prod_{i=1}^{p} \theta\left(l_{i}, l_{q}\right), \quad S_{p}=\sum_{j=1}^{p} k^{j} \prod_{i=1}^{p} \theta\left(l_{i}, l_{q}\right)
$$

Then, for $q>p$, the above can be written as

$$
d_{\theta}\left(l_{p}, l_{q}\right) \preceq d_{\theta}\left(l_{o}, l_{1}\right)\left[S_{q-1}-S_{P}\right]
$$




$$
\left|d_{\theta}\left(l_{p}, l_{q}\right)\right| \leq\left|d_{\theta}\left(l_{o}, l_{1}\right)\right|\left[S_{q-1}-S_{p}\right]
$$

Now, by taking $p \rightarrow \infty$ in the above expression, we get

$$
\left|d_{\theta}\left(l_{p}, l_{q}\right)\right| \rightarrow 0 .
$$

By Lemma 2.4, $\left\{l_{p}\right\}$ is a Cauchy sequence in $\mathbb{G}$, which is complete, so there exists some $r \in \mathbb{G}$ such that limit $_{p \rightarrow \infty} l_{p}=r$. We now show that $r \in \mathrm{S} r$ and $r \in \mathbb{T} r$. From (6.4), we have

$$
\begin{aligned}
& \lambda_{1} d_{\theta}\left(l_{2 p}, r\right)+\lambda_{2} \frac{d_{\theta}\left(l_{2 p}, \mathrm{~S} l_{2 p}\right) d_{\theta}(r, \mathbb{T} r)}{1+d\left(l_{2 p}, r\right)}+\lambda_{3} \frac{\left.d_{\theta}\left(r, \mathbb{S} l_{2 p}\right) d_{1} l_{2 p}, \mathbb{T} r\right)}{1+d_{\theta}\left(l_{2 p}, r\right)} \\
& +\lambda_{4} \frac{d_{\theta}\left(l_{2 p}, \mathbb{S} l_{2 p}\right) d_{\theta}\left(l_{2 p}, \mathbb{T} r\right)}{\left.1+d_{(} l_{2 p}, r\right)}+\lambda_{5} \frac{d_{\theta}\left(r, S l_{2 p}\right) d_{\theta}(r, \mathbb{T} r)}{1+d_{\theta}\left(l_{2 p}, r\right)} \in s\left(\mathbb{S} l_{2 p}, \mathbb{T} r\right), \\
& \lambda_{1} d_{\theta}\left(l_{2 p}, r\right)+\lambda_{2} \frac{d_{\theta}\left(l_{2 p}, S l_{2 p}\right) d_{\theta}(r, \mathbb{T} r)}{1+d\left(l_{2 p}, r\right)}+\lambda_{3} \frac{\left.d_{\theta}\left(r, S l_{2 p}\right) d_{(} l_{2 p}, \mathbb{T} r\right)}{1+d_{\theta}\left(l_{2 p}, r\right)} \\
& +\lambda_{4} \frac{d_{\theta}\left(l_{2 p}, \mathbb{S} l_{2 p}\right) d_{\theta}\left(l_{2 p}, \mathbb{T} r\right)}{\left.1+d_{(} l_{2 p}, r\right)}+\lambda_{5} \frac{d_{\theta}\left(r, S l_{2 p}\right) d_{\theta}(r, \mathbb{T} r)}{1+d_{\theta}\left(l_{2 p}, r\right)} \in \bigcap_{a \in \mathbb{S} l_{2 p}} s(a, \mathbb{T} r) .
\end{aligned}
$$

Since $l_{2 p+1} \in S l_{2 p}$, we get

$$
\begin{gathered}
\lambda_{1} d_{\theta}\left(l_{2 p}, r\right)+\lambda_{2} \frac{d_{\theta}\left(l_{2 p}, \mathbb{S} l_{2 p}\right) d_{\theta}(r, \mathbb{T} r)}{1+d\left(l_{2 p}, r\right)}+\lambda_{3} \frac{\left.d_{\theta}\left(r, \mathbb{S} l_{2 p}\right) d_{(} l_{2 p}, \mathbb{T} r\right)}{1+d_{\theta}\left(l_{2 p}, r\right)} \\
+\lambda_{4} \frac{d_{\theta}\left(l_{2 p}, S l_{2 p}\right) d_{\theta}\left(l_{2 p}, \mathbb{T} r\right)}{\left.1+d_{(} l_{2 p}, r\right)}+\lambda_{5} \frac{d_{\theta}\left(r, S l_{2 p}\right) d_{\theta}(r, \mathbb{T} r)}{1+d_{\theta}\left(l_{2 p}, r\right)} \in s\left(l_{2 p+1}, \mathbb{T} r\right), \\
\lambda_{1} d_{\theta}\left(l_{2 p}, r\right)+\lambda_{2} \frac{d_{\theta}\left(l_{2 p}, S l_{2 p}\right) d_{\theta}(r, \mathbb{T} r)}{1+d\left(l_{2 p}, r\right)}+\lambda_{3} \frac{\left.d_{\theta}\left(r, \mathbb{S} l_{2 p}\right) d_{(} l_{2 p}, \mathbb{T} r\right)}{1+d_{\theta}\left(l_{2 p}, r\right)} \\
+\lambda_{4} \frac{d_{\theta}\left(l_{2 p}, \mathbb{S} l_{2 p}\right) d_{\theta}\left(l_{2 p}, \mathbb{T} r\right)}{\left.1+d_{(} l_{2 p}, r\right)}+\lambda_{5} \frac{d_{\theta}\left(r, S l_{2 p}\right) d_{\theta}(r, \mathbb{T} r)}{1+d_{\theta}\left(l_{2 p}, r\right)} \in \bigcup_{b \in \mathbb{T} r} s\left(d_{\theta}\left(l_{2 p+1}, b\right)\right)
\end{gathered}
$$

So, there exists some $r_{p} \in \mathbb{T} r$ such that

$$
\begin{aligned}
& \lambda_{1} d_{\theta}\left(l_{2 p}, r\right)+\lambda_{2} \frac{d_{\theta}\left(l_{2 p}, \mathrm{~S} l_{2 p}\right) d_{\theta}(r, \mathbb{T} r)}{1+d\left(l_{2 p}, r\right)}+\lambda_{3} \frac{\left.d_{\theta}\left(r, S l_{2 p}\right) d_{(} l_{2 p}, \mathbb{T} r\right)}{1+d_{\theta}\left(l_{2 p}, r\right)} \\
& +\lambda_{4} \frac{d_{\theta}\left(l_{2 p}, \mathrm{~S} l_{2 p}\right) d_{\theta}\left(l_{2 p}, \mathbb{T} r\right)}{\left.1+d_{(} l_{2 p}, r\right)}+\lambda_{5} \frac{d_{\theta}\left(r, \mathrm{~S} l_{2 p}\right) d_{\theta}(r, \mathbb{T} r)}{1+d_{\theta}\left(l_{2 p}, r\right)} \in s\left(d_{\theta}\left(l_{2 p+1}, r_{p}\right)\right) .
\end{aligned}
$$

Therefore, by definition, we have

$$
\begin{aligned}
d_{\theta}\left(l_{2 p+1}, r_{p}\right) & \preceq \lambda_{1} d_{\theta}\left(l_{2 p}, r\right)+\lambda_{2} \frac{d_{\theta}\left(l_{2 p}, S l_{2 p}\right) d_{\theta}(r, \mathbb{T} r)}{1+d\left(l_{2 p}, r\right)}+\lambda_{3} \frac{\left.d_{\theta}\left(r, S l_{2 p}\right) d_{(} l_{2 p}, \mathbb{T} r\right)}{1+d_{\theta}\left(l_{2 p}, r\right)} \\
& +\lambda_{4} \frac{d_{\theta}\left(l_{2 p}, S l_{2 p}\right) d_{\theta}\left(l_{2 p}, \mathbb{T} r\right)}{\left.1+d_{(} l_{2 p}, r\right)}+\lambda_{5} \frac{d_{\theta}\left(r, S l_{2 p}\right) d_{\theta}(r, \mathbb{T} r)}{1+d_{\theta}\left(l_{2 p}, r\right)} .
\end{aligned}
$$

Using the g.l.b property of $\mathbb{T}$, we obtain

$$
d_{\theta}\left(l_{2 p+1}, r_{p}\right) \preceq \lambda_{1} d_{\theta}\left(l_{2 p}, r\right)+\lambda_{2} \frac{d_{\theta}\left(l_{2 p}, l_{2 p+1}\right) d_{\theta}\left(r, r_{p}\right)}{1+d\left(l_{2 p}, r\right)}+\lambda_{3} \frac{\left.d_{\theta}\left(r, l_{2 p+1}\right) d_{1} l_{2 p}, r_{P}\right)}{1+d_{\theta}\left(l_{2 p}, r\right)}
$$




$$
+\lambda_{4} \frac{d_{\theta}\left(l_{2 p}, l_{2 p+1}\right) d_{\theta}\left(l_{2 p}, r_{p}\right)}{\left.1+d_{(} l_{2 p}, r\right)}+\lambda_{5} \frac{d_{\theta}\left(r, l_{2 p+1}\right) d_{\theta}\left(r, r_{p}\right)}{1+d_{\theta}\left(l_{2 p}, r\right)} .
$$

By definition 6.4, we get

$$
d_{\theta}\left(r, r_{p}\right) \preceq \theta\left(r, r_{p}\right)\left[d_{\theta}\left(r, l_{2 p+1}\right)+d_{\theta}\left(l_{2 p+1}, r_{p}\right)\right] .
$$

Thus,

$$
\begin{aligned}
d_{\theta}\left(l_{2 p+1}, r_{p}\right) & \preceq \theta\left(r, r_{p}\right) d_{\theta}\left(r, l_{2 p+1}\right)+\theta\left(r, r_{p}\right) \lambda_{1} d_{\theta}\left(l_{2 p}, r\right)+\theta\left(r, r_{p}\right) \lambda_{2} \frac{d_{\theta}\left(l_{2 p}, l_{2 p+1}\right) d_{\theta}\left(r, r_{p}\right)}{1+d\left(l_{2 p}, r\right)} \\
& +\theta\left(r, r_{p}\right) \lambda_{3} \frac{\left.d_{\theta}\left(r, l_{2 p+1}\right) d_{(} l_{2 p}, r_{P}\right)}{1+d_{\theta}\left(l_{2 p}, r\right)}+\theta\left(r, r_{p}\right) \lambda_{4} \frac{d_{\theta}\left(l_{2 p}, l_{2 p+1}\right) d_{\theta}\left(l_{2 p}, r_{p}\right)}{\left.1+d_{(} l_{2 p}, r\right)} \\
& +\theta\left(r, r_{p}\right) \lambda_{5} \frac{d_{\theta}\left(r, l_{2 p+1}\right) d_{\theta}\left(r, r_{p}\right)}{1+d_{\theta}\left(l_{2 p}, r\right)} \\
\left|d_{\theta}\left(l_{2 p+1}, r_{p}\right)\right| \leq & \theta\left(r, r_{p}\right)\left|d_{\theta}\left(r, l_{2 p+1}\right)\right|+\theta\left(r, r_{p}\right) \lambda_{1}\left|d_{\theta}\left(l_{2 p}, r\right)\right|+\theta\left(r, r_{p}\right) \lambda_{2}\left|\frac{d_{\theta}\left(l_{2 p}, l_{2 p+1}\right) d_{\theta}\left(r, r_{p}\right)}{1+d\left(l_{2 p}, r\right)}\right| \\
& +\theta\left(r, r_{p}\right) \lambda_{3}\left|\frac{\left.d_{\theta}\left(r, l_{2 p+1}\right) d_{(} l_{2 p}, r_{P}\right)}{1+d_{\theta}\left(l_{2 p}, r\right)}\right|+\theta\left(r, r_{p}\right) \lambda_{4}\left|\frac{d_{\theta}\left(l_{2 p}, l_{2 p+1}\right) d_{\theta}\left(l_{2 p}, r_{p}\right)}{\left.1+d_{(} l_{2 p}, r\right)}\right| \\
& +\theta\left(r, r_{p}\right) \lambda_{5}\left|\frac{d_{\theta}\left(r, l_{2 p+1}\right) d_{\theta}\left(r, r_{p}\right)}{1+d_{\theta}\left(l_{2 p}, r\right)}\right| .
\end{aligned}
$$

Taking limit as $p \rightarrow \infty$ in the above inequality, we get

$$
\left|d_{\theta}\left(l_{2 p+1}, r_{p}\right)\right| \rightarrow 0 .
$$

By Lemma 2.4, $r_{p} \rightarrow r$ as $p \rightarrow \infty$. Further, since $\mathbb{T} r$ is closed, then $r \in \mathbb{T} r$. Similarly, $r \in \mathbb{S} r$. Thus, $\mathbb{S}$ and $\mathbb{T}$ have a common fixed point.

\section{Conclusion}

In this paper, we studied common fixed point results for almost contraction, Banach and Kannan type contractions in the setting of complex-valued extended $b$-metric spaces. Starting from the notion of complex-valued metric spaces, our results complement several significant fixed point theorems of $b$-metric and extended $b$-metric spaces in the frame of crisp mappings. We hope that our presented idea herein will be a source of motivation for other researchers to extend and improve these results suitable for their applications.

\section{Competing Interests}

The authors declare that they have no competing interests.

\section{Acknowledgement}

The authors are grateful to the editors and the anonymous referee(s) for careful checking of the details and for their helpful comments to improve this paper. 


\section{References}

[1] J. Ahmad, C. Klin-Eam, and A. Azam, (2013). Common fixed points for multivalued mappings in complex valued metric spaces with applications. In Abstract and Applied Analysis (Vol. 2013). Hindawi.

[2] J. Ahmad, N. Hussain, A. Azam and M. Arshad (2015). Common fixed point results in complex valued metric with application to system of integral equations. Journal of Nonlinear and Convex Analysis, 29(5), 855-871.

[3] M. Aiman (2014). Some common fixed point theorems in complex valued b-metric spaces. The Scientific World Journal, 2014.

[4] S. Aleksić, Z. Kadelburg, Z. D. Mitrovic and S. Radenovic (2019). A new survey: Cone metric spaces, Journal of the International Mathematical Virtual Institute, Vol.9, 93-121.

[5] A. Azam, B. Fisher and M. Khan (2011). Common fixed point theorems in complex valued metric spaces. Numerical Functional Analysis and Optimization, 32(3), 243-25.

[6] A. Azam, J. Ahmad and P. Kumam (2012). Common fixed point theorems for multi-valued mappings in complex-valued metric spaces. Numer. Funct. Anal. Optim. 33(5), 590-600.

[7] A. Azam, J. Ahmad, and P. Kumam (2013). Common fixed point theorems for multi-valued mappings in complex-valued metric spaces. Journal of Inequalities and Applications, 2013(1), 578.

[8] A. Azam,and N. Mehmood (2013). Multivalued fixed point theorems in tvs-cone metric spaces. Fixed Point Theory and Applications 2013, no. 1, 184.

[9] I. A. Bakhtin (1989). The contraction principle in quasimetric spaces. Functional Analysis, vol. 30, 26 - 37.

[10] S. Banach (1922). Sur les operation dans les ensembles abstraits et applications aux equations integrales. Fund. Math. (French), 133-181.

[11] S. Bhatt, S. Chaukiyal and R. C. Dimiri (2011). Common fixed point mappings satisfying rational inequality in complex valued metric spaces. International journal of Pure and Applied Mathematics. 73 (2), 159-164.

[12] M. Berinde, and V. Berinde (2007). On a general class of multi-valued weakly Picard mappings. Journal of Mathematical Analysis and Applications, 326(2), 772-782.

[13] S. K. Chatterjea (1972). Fixed point theorem. C.R. Acad. Bulgare Sci. 25, 727-730.

[14] A. K. Dubey, R. Shukla and R. P. Dubey (2015). Some fixed point theorems in complex valued b-metric spaces. Journal of Complex Systems, 2015 (2015).

[15] A. K. Dubey (2015). Common fixed point results for contractive mappings in complex valued b-metric spaces. Nonlinear Functional Analysis and Applications, vol. 20, 257- 268.

[16] A. K. Dubey (2016). Complex Valued-Metric Spaces and Common Fixed Point Theorems under Rational Contractions. Journal of Complex analysis, 2016.

[17] M. Edelstein (1962). On fixed and periodic points under contractive mappings. Journal of the London Mathematical Society, 1(1), 74-79.

[18] L. G. Huang, and X. Zhang (2007). Cone metric spaces and fixed point theorems of contractive mappings. Journal of mathematical Analysis and Applications, 332(2), 1468-1476.

[19] S. Jankovic, Z. Golubovic, and S. Radenovic (2010). Compatible and weakly compatible mappings in cone metric spaces. Mathematical and Computer Modelling, 52(9-10), 1728-1738.

[20] T. Kamran, M. Samreen, Q., UL Ain, (2017). A generalization of $b$-metric space and some fixed point theorems. Mathematics, 5(2), 19.

[21] J. Kumar (2015). Common Fixed Point Theorem for Generalized Contractive Type Maps on Complex Valued b-Metric Spaces. International Journal of Mathematical Analysis Vol. 9, 2327 - 2334.

[22] N. Mizoguchi, and W. Takahashi (1989). Fixed point theorems for multivalued mappings on complete metric spaces. Journal of Mathematical Analysis and Applications, 141(1), 177-188.

[23] S. B. Nadler, (1969). Multi-valued contraction mappings. Pacific Journal of Mathematics, 30(2), 475-488.

[24] B .E. Rhoades (1977). A comparison of various definitions of contractive mappings. Transactions of the American Mathematical Society, 226, 257-290.

[25] P. Rao, R. Swamy, and J. R. Prasad (2013). A common fixed point theorem in complex valued b-metric spaces. Bulletin of Mathematics and Statistics Research, Vol. 1, 1-8. 
[26] W. Shatanawi, V. C. Rajic, S. Radenovic, and A. Al-Rawashdeh (2012). Mizoguchi-Takahashi-type theorems in tvs-cone metric spaces. Fixed Point Theory and Applications, 2012(1), 106. 